\title{
Condensación de Bose-Einstein en un campo gravitacional homogéneo
}

Tesis que presenta:

Fís. Juan Israel Rivas Sánchez

Para obtener el grado de:

Maestro en Ciencias (Física)

Asesor:

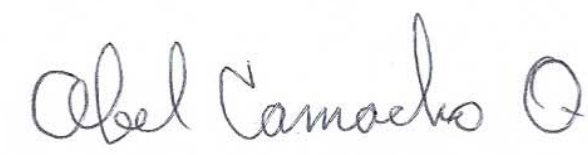

Dr. Abel Camacho Quintana

Profesor Titular C

Departamento de Física

México, D. F., Marzo 2010. 


\section{AGRADECIMIENTOS}

Deseo expresar mi agradecimiento a las personas que me apoyaron en la realización de esta tesis tanto de manera personal como académica. Agradezco a mi madre y agradezco a mi esposa Imelda por su comprensión y paciencia, y demás familiares y amigos.

Agradezco al Dr. Abel Camacho Quintana por haber fungido como asesor de esta tesis.

Agradezco el Apoyo recibido por CONACyT, a través del programa nacional de becas de posgrado 160453, sin el no hubiera sido posible realizar los estudios de maestría y este trabajo de investigación. 



\section{Contenido}

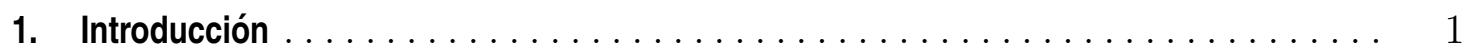

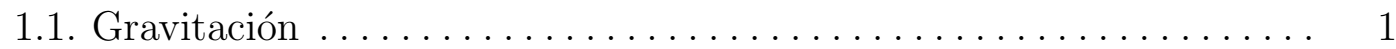

1.1.1. Principio de Equivalencia Débil . . . . . . . . . . . . . . . 1

1.1.2. Invariancia Local de Lorentz . . . . . . . . . . . . . . . . . . . . 10

1.1.3. Invariancia Local de la Posición. . . . . . . . . . . . . . . . . . 13

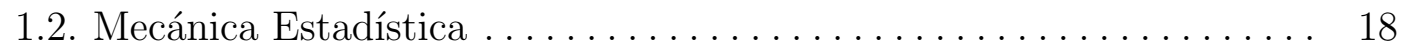

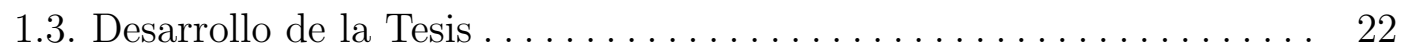

2. Fundamentos de la teoría de la relatividad general $\ldots \ldots \ldots \ldots \ldots \ldots \ldots \ldots$

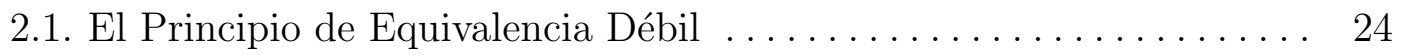

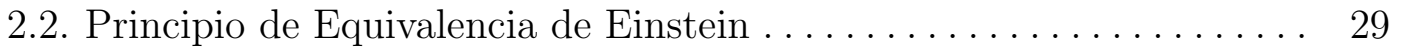

3. Condensación de Bose Einstein en ausencia de campos gravitacionales y fuerzas

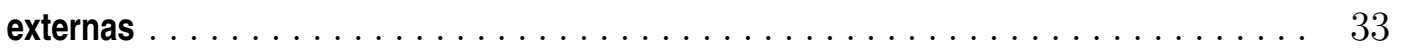

4. Condensación de Bose Einstein en un campo gravitacional homogéneo . . . . . . . . 47 
4.1. Gas ideal de Bose-Einstein confinado en un recipiente. . . . . . . . . . . 48

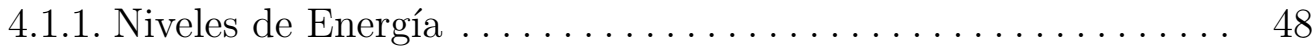

4.1.2. Temperatura de condensación en un campo gravitacional

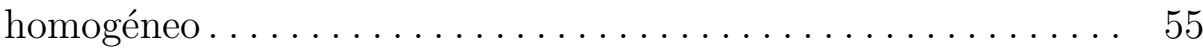

4.1.3. Presión de un gas de Bose-Einstein en un campo gravitacional homogéneo . . . . . . . . . . . . . . . . . . . . . 60

4.2. Gas ideal de Bose-Einstein confinado por osciladores armónicos

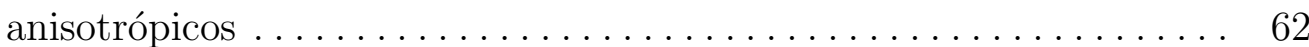

5. Conclusiones y Perspectivas $\ldots \ldots \ldots \ldots \ldots \ldots \ldots \ldots \ldots \ldots \ldots \ldots \ldots \ldots$

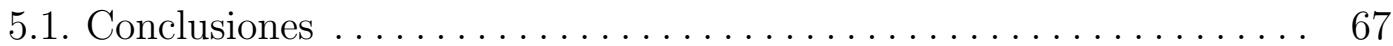

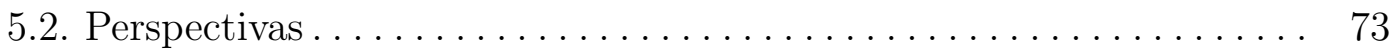

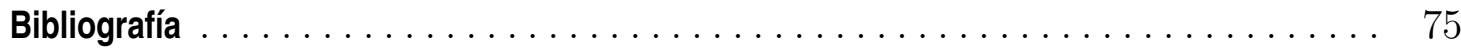

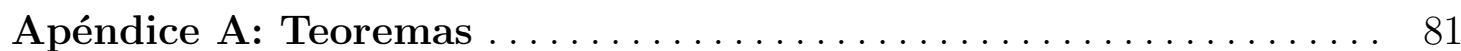

A.1. Teoremas ..................................... 81

A.1.1.Teorema de Convergencia Absoluta. . . . . . . . . . . . . . . . . 81

A.1.2. Teorema de sucesiones crecientes . . . . . . . . . . . . . . . 81

Apéndice B: Teoría de Perturbaciones independiente del tiempo. . . 83

B.1. Teoría de Perturbaciones independiente del tiempo . . . . . . . . . 83

B.1.1. El Problema de dos estados . . . . . . . . . . . . . . . . . . . . 85

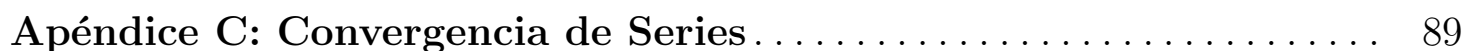


CAPÍtulo 1

\section{Introducción}

\subsection{Gravitación}

\subsubsection{Principio de Equivalencia Débil}

El Principio de Equivalencia Débil ha jugado un papel muy importante para el desarrollo de la teoría gravitacional. En 1907, Einstein usó este principio como una base fundamental para desarrollar su teoría. Hoy en día observamos el Principio de Equivalencia Débil como una de las bases de la Relatividad General o Gravedad No-Newtoniana, pero la idea más importante es la de considerar el espacio-tiempo como una variedad curva.

El Principio de Equivalencia Débil (PED) se puede enunciar de la siguiente forma: si un cuerpo de prueba sin carga eléctrica es puesto en un evento inicial en el espacio-tiempo y después se le da una velocidad inicial, entonces su trayectoria subsecuente será independiente de su estructura interna y composición [1].

Galileo ya había observado que en el campo gravitacional de la tierra, todos los cuerpos caen con la misma aceleración [2], hecho conocido como la Universalidad de Caída Libre. Podemos señalar que es una consecuencia y a su vez una prueba 
del PED. Esto supone una relación entre dos conceptos diferentes, la masa inercial $m_{i}$ y la masa gravitacional pasiva $m_{p}$. En efecto, la ecuación de movimiento clásica para una partícula puntual inmersa en un campo gravitacional homogéneo (a lo largo de la dirección z) es:

$$
m_{i} \frac{d^{2} \vec{r}}{d t^{2}}=-m_{p} g \widehat{z}
$$

PED establece que la solución de esta ecuación depende de sus condiciones iniciales, pero no de su estructura interna o composición de la partícula. En otras palabras, está basado en el hecho de que

$$
\frac{m_{i}}{m_{p}}=\alpha
$$

donde $\alpha$ es una constante universal, es decir, la misma para todas las partículas. Hay que hacer dos observaciones. La primera observación, PED requiere como una condición necesaria que el valor de $\alpha$ sea independiente de la partícula. En otras palabras, el valor particular de $\alpha$ es completamente irrelevante $(\alpha \neq 0)$. Esto puede entenderse al introducir el valor de $\alpha$ en la ecuación (1.2)

$$
\frac{d^{2} \vec{r}}{d t^{2}}=-\widetilde{g} \widehat{z}
$$

Aquí $\widetilde{g}=\frac{g}{\alpha}$. Un valor de $\alpha \neq 1$ podría implicar una redefinición de la aceleración de la gravedad. Si el campo gravitacional no es homogéneo tenemos,

$$
m_{i} \frac{d^{2} \vec{r}}{d t^{2}}=-m_{p} \nabla \phi
$$

donde $\phi=-\frac{G m_{a}}{r}, m_{a}$ es la masa gravitacional activa, $G$ es la constante universal de la gravitación. Si usamos la ecuación (1.2) en la expresión donde el campo gravitacional ya no es homogéneo, obtenemos

$$
\frac{d^{2} \vec{r}}{d t^{2}}=-\widetilde{G} m_{a} \nabla\left(\frac{1}{r}\right),
$$


donde $\widetilde{G}$ es $\widetilde{G}=\frac{G}{\alpha}$. En un experimento con campo gravitacional homogéneo no se pueden detectar $g$ o $\alpha$, solo la aceleración efectiva de la gravedad, es decir, $\widetilde{g}$. El valor de $\alpha$ es irrelevante (asumiendo que PED es válido), no puede ser medido (en cuatro dimensiones). La segunda observación es que para geometrizar a la gravedad se requiere que PED se cumpla. En otras palabras, si $\alpha$ dependiera de la partícula, entonces ya no se cumpliría PED y, por lo tanto, la geometrización de la gravedad sería una cuestión discutible, suponiendo una variedad tetra-dimensional. Por último, debemos añadir que toda prueba experimental de la validez o invalidez de PED, requiere el uso de más de una partícula. De hecho, PED se basa en el carácter universal de $\alpha$ y una partícula no puede darnos este tipo de información.

La versión matemática de PED establece: El movimiento de una partícula sin carga eléctrica está determinada por la geodésica de la variedad correspondiente [1]. Nótese que detrás de esta frase yace el concepto de trayectoria. La verificación experimental de PED a nivel clásico se ha realizado en diferentes aproximaciones y con diferentes dispositivos [3], pero la versión mecánico-cuántica demanda un análisis más cuidadoso. Esta última observación adquiere relevancia ya que en la teoría cuántica el concepto de trayectoria no es admisible, es decir, la teoría puede formularse sin el [4].

PED es válido para todas las clases de materia (materia de bulto macroscópica, átomos, materia neutra y cargada). Por lo tanto, las pruebas experimentales del PED se han hecho con todo tipo de materia. Sin embargo, las pruebas más precisas se han obtenido con materia neutra.

Una verificación directa de PED se obtiene al comparar la aceleración de dos cuerpos de prueba de diferente composición en una campo gravitacional externo. Si el principio fuera violado, entonces las aceleraciones de los cuerpos serían difer- 
entes. La masa inercial $m_{i}$ de un cuerpo de prueba típico se puede componer de varios tipos de masa-energía: energía en reposo, energía electromagnética, energía de interacción débil, etcétera. Si una de estas formas de energía contribuye a la masa gravitacional pasiva $m_{p}$ de una forma diferente de lo que lo hace con la masa inercial $m_{i}$, podría resultar en una violación al PED [1]. Podemos entonces escribir

$$
m_{p}=m_{i}+\sum_{A} \eta^{A} E^{A} / c^{2}
$$

donde $E^{A}$ es la energía interna del cuerpo generado por la interacción $A$, y $\eta^{A}$ es un parámetro sin dimensiones que mide la magnitud de la violación de PED inducida por la interacción, y $c$ es la velocidad de la luz. Para dos cuerpos de masas diferentes, la aceleración está dada por

$$
a_{1}=\left(1+\sum_{A} \eta^{A} E^{A} / m_{1} c^{2}\right) g, \quad a_{2}=\left(1+\sum_{A} \eta^{A} E^{A} / m_{2} c^{2}\right) g
$$

donde la $a_{1}$ y $a_{2}$ son las aceleraciones de los cuerpos de masas $m_{1}$ y $m_{2}$, respectivamente.

Una medida o límite sobre la diferencia relativa en la aceleración aparece en una cantidad llamada la "fracción de Eötvös" dada por [1]

$$
\eta \equiv \frac{2\left|a_{1}-a_{2}\right|}{\left|a_{1}+a_{2}\right|}=\sum_{A} \eta^{A}\left(\frac{E_{1}^{A}}{m_{1} c^{2}}-\frac{E_{2}^{A}}{m_{2} c^{2}}\right) .
$$

Así, el límite experimental sobre $\eta$ pone límites a los parámetros $\eta^{A}$ que nos indicarían una violación del PED.

Otra forma de definir el parámetro de Eötvös es [5]:

$$
\eta \leq 2\left|\frac{\left(m_{i} / m_{p}\right)_{a}-\left(m_{i} / m_{p}\right)_{b}}{\left(m_{i} / m_{p}\right)_{a}+\left(m_{i} / m_{p}\right)_{b}}\right|,
$$


para dos masas de prueba de diferente composición denotadas por el subíndice $a$ y $b$, con masa inercial $m_{i a}$ y $m_{i b}$, así como masa gravitacional $m_{p a}$ y $m_{p b}$.

Históricamente son cuatro los métodos que ponen a prueba a nivel clásico PED con materia neutra:

1. El método del péndulo de Newton.

Los experimentos para poner a prueba PED fueron realizados por primera vez de manera más formal por Isaac Newton. En los Principia Newton describe el experimento del péndulo, donde se usan dos masas de diferente composición ( $a$ y $b$ ); en un péndulo se comparan sus periodos $T_{a, b}=2 \pi \sqrt{\left(m_{i} / m_{p}\right)_{a, b} /(l / g)}$. Si la longitud del péndulo $l$ y el campo gravitacional $g$ en laboratorio es la misma para todos los experimentos, cualquier diferencia de periodos podría indicar una violación de PED. La diferencia de periodos está dada por la siguiente relación:

$$
2 \frac{T_{a}^{2}-T_{b}^{2}}{T_{a}^{2}+T_{b}^{2}}=2\left|\frac{\left(m_{i} / m_{p}\right)_{a}-\left(m_{i} / m_{p}\right)_{b}}{\left(m_{i} / m_{p}\right)_{a}+\left(m_{i} / m_{p}\right)_{b}}\right|=\eta
$$

Newton mostró con su experimento el valor del parámetro de Eötvös con una precisión de $10^{-3}$ [6]. Después, Friedrich Wilhelm Bessel en 1827, usando el método del péndulo mejoró la precisión a $\eta \leq 10^{-5}$ [7]. La precisión más alta fué obtenida por H.H. Potter en 1927, quien obtuvo una precisión de $\eta \leq 10^{-6}$ [8]. La precisión de experimentos llevados a cabo con el péndulo es limitada. La razón es que para medir el periodo del péndulo uno necesita realizar la aproximación $\operatorname{sen} x=x$ en la ecuación diferencial de segundo orden no-lineal. Para la prueba de precisión, esta aproximación conduce a errores sistemáticos, pues el periodo del péndulo ya no es independiente de la amplitud la cual disminuye con el tiempo de integración. 
2. El método de la balanza de torsión de Eötvös.

El experimento clásico más preciso fue realizado por R. V. Eötvös, llevado a cabo con una balanza de torsión. El experimento puede ser descrito de la siguiente manera: Dos objetos de diferente composición están conectados por una barra de longitud $r$, y suspendidos en una orientación horizontal por un alambre fino. Si la aceleración gravitacional de los cuerpos es diferente, habrá una torsión inducida $\mathbf{N}$ sobre el alambre, dada por

$$
\mathbf{N}=\eta r\left(\mathbf{g} \times \widehat{\mathbf{e}}_{\mathbf{w}}\right) \cdot \widehat{\mathbf{e}}_{\mathbf{r}}
$$

donde $\eta$ esta dado por la ecuación (1.8), g es la aceleración gravitacional, y $\widehat{\mathbf{e}}_{\mathbf{w}} \mathrm{y}$ $\widehat{\mathbf{e}}_{\mathbf{r}}$ son vectores unitarios a lo largo del alambre y de la barra, respectivamente. Si todo el aparato es rotado alrededor de $\mathbf{w}$ con una velocidad angular $|\mathbf{w}|$, la torsión estaría modulada por un periodo $2 \pi / w$, ver Fig. 1.1. Las balanzas de torsión pueden operar de dos modos: (i) En el modo solar se usa el campo gravitacional del sol donde la fuerza gravitacional que actúa sobre las masas de prueba es equilibrada por la fuerza centrífuga debido a la rotación de la tierra. (ii) En el modo terrestre la balanza de torsión gira alrededor del eje del alambre de manera sincronizada a la rotación de la Tierra. Debido a la rotación de la Tierra, el vector de gravedad en la superficie de la Tierra está inclinado en un pequeño ángulo respecto a la vertical. Por lo tanto, un desequilibrio de la fuerza inercial y gravitacional resultaría en una variación periódica de este ángulo. En los experimentos del Baron Roland von Eötvös, g es la aceleración de la gravedad de la Tierra y el aparato fué rotado en la dirección del alambre. Sus primeras series de trabajos publicadas en 1890, establecen la constancia de la aceleración gravitacional con una precisión de $\eta \leq 10^{-8}$ [9]. Después, con V. 


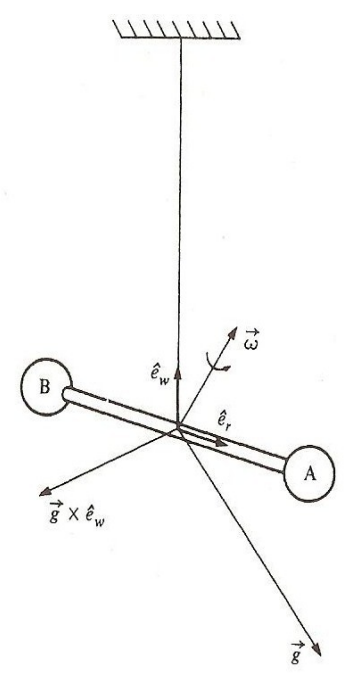

Fig. 1.1. Esquema de la balanza de torsión de Eötvös, donde $\mathbf{g}$ es la aceleración gravitacional externa, w es el vector velocidad alrededor del cual el aparato va ser rotado, y $\widehat{\mathbf{e}}_{\mathbf{w}} \mathrm{y} \widehat{\mathbf{e}}_{\mathbf{r}}$ son vectores unitarios a lo largo del alambre y de la barra, respectivamente [1].

Pekar y E. Fekete se incrementó la precisión a $\eta \leq 10^{-9}$ [10]. Hoy en día las precisiones más altas obtenidas al usar la balanza de torsión en el modo terrestre se le atribuyen a Adelberger y colaboradores en la Universidad de Washington en Seattle con la mejoría de resultados desde 1987 [11, 12]. Los resultados varían para diferentes materiales entre $\eta=(-1.9 \pm 2.5) \times 10^{-12}$ por la comparación del berilio y el cobre y $\eta=(-0.2 \pm 2.8) \times 10^{-12}$ por la comparación de berilio y aluminio [13]. En experimentos tipo solar, Dicke y sus colaboradores [14], y Braginsky [15], determinaron $\eta=(1.3 \pm 1.0) \times 10^{-11}$ para el oro y aluminio y $\eta=(-0.3 \pm 0.9) \times 10^{-12}$ para plata y aluminio, respectivamente. Las principales fuentes de error en estos experimentos son el ruido sísmico y el acoplamiento de la balanza de torsión con los gradientes en el campo gravitacional externo (producidos, por ejemplo, por los experimentos). Los intentos de mejorar estos 
resultados se han centrado en las diferentes formas de suspensión de las masas, incluyendo levitación magnética, flotación sobre líquidos y caída libre en orbitas.

3. El método de Caída Libre de Galileo.

En 1648, Galileo Galilei escribió en su famoso "Discorsi et dimonstrazioni matematiche intorno a due nuove scienze" [2]: El experimento fué realizado con dos cuerpos de diferente peso, dejándolos caer desde la torre inclinada de Pisa para observar si caen con la misma velocidad.

El "STEP" (Satellite Test of the Equivalence Principle) por sus siglas en inglés, es un satélite que intenta probar PED con una precisión de $10^{-18}$. El concepto más simple de STEP es el experimento de Galileo, con la diferencia de que la masas de pruebas caen todas alrededor de la tierra. Si hay una diferencia en la aceleración las masas se irán en orbitas separadas y en un tiempo corto las masas se separarían en proporción al cuadrado del tiempo de caída. Para tiempos de una orbita o más, las masas tenderían a regresar a sus posiciones iniciales, con una desviación secular. La ventaja de STEP con respecto a cualquier experimento que se realice en la Tierra, es que el experimento se repite periódicamente, para más información sobre STEP ver [16].

Experimentos modernos de Caída Libre desarrollados por grupos de Estados Unidos, Italia, y Japón dan resultados entre 1 y 3 partes en $10^{10}$.

4. El método celeste de Newton ( el cual consiste en usar las observaciones del sistema Tierra-Luna o de las Lunas de Júpiter en el campo gravitacional del sol).

El método celeste fué refinado por Laplace en 1798 y reinventado en 1960 en el contexto de mediciones láser a satélites. El límite más preciso obtenido a la fecha reportado por Dicke es de 5 partes en $10^{13}$, ver la Fig. 1.2 . 


\section{TESTS OF THE \\ WEAK EQUIVALENCE PRINCIPLE}

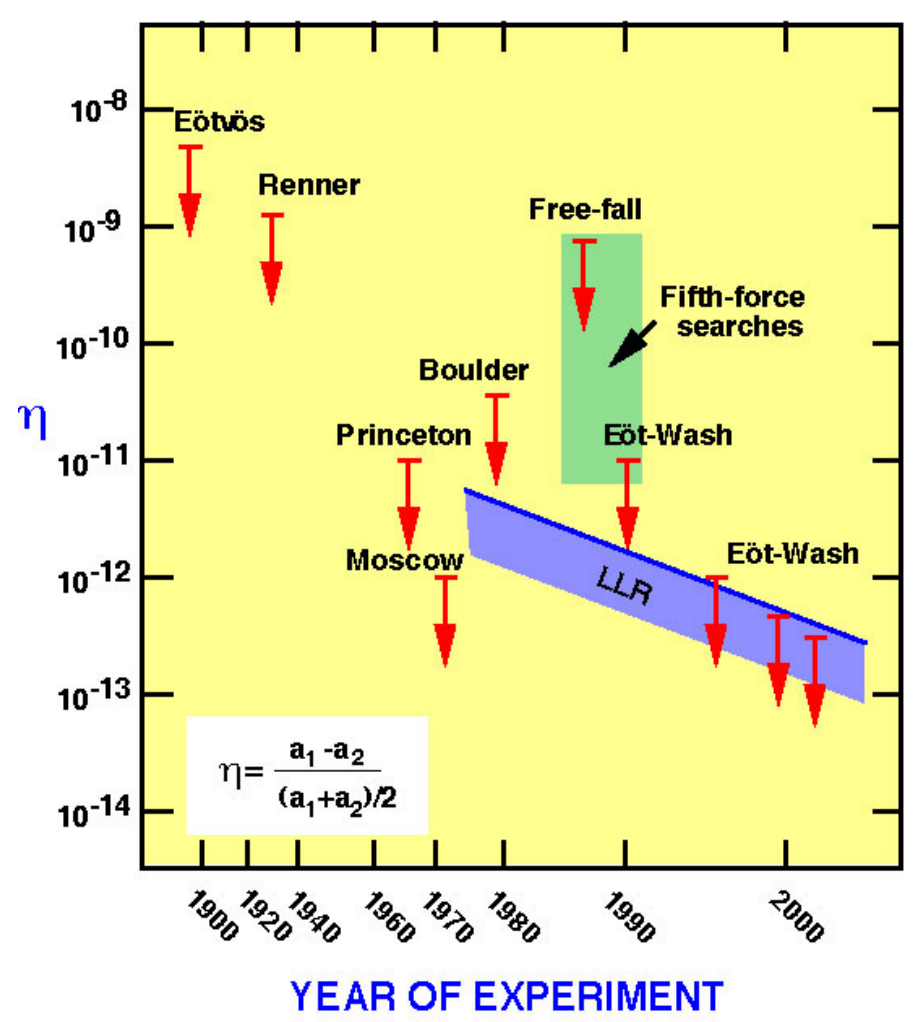

Fig. 1.2. Algunas pruebas experimentales del PED que muestran el límite sobre el parámetro $\eta$, el cual mide la diferencia fraccional de la aceleración de diferentes cuerpos materiales. Los experimentos de caída libre y los experimentos de Eöt-Wash estaban originalmente desarrollados para buscar una quinta fuerza (región en verde). La banda en azul muestra limites sobre el parámetro $\eta$ para cuerpos gravitando desde el Lunar Láser Ranging (LLR por sus siglas en inglés) [17.

En la literatura uno encuentra varios términos, como el Principio de Equivalencia Débil (PED), Principio de Equivalencia Fuerte (PEF), y el Principio de Equivalencia de Einstein (PEE). Pero hay que entender el significado que implica cada uno de estos Principios. Como ya se ha mencionado PED no es más que la 
Universalidad de Caída Libre de los cuerpos. PEF está comprendido por la Invariancia Local de Lorentz (ILL) y la Invariancia Local de la Posición (ILP). ILL se define como el resultado de cualquier prueba experimental local no-gravitacional la cual es independiente de la velocidad del aparato en caída libre. ILP se define como el resultado de cualquier prueba experimental local no-gravitacional la cual es independiente de donde y cuando en el universo sea realizado el experimento. Por ultimo, PEE exige la validez de PED y PEF [1].

\subsubsection{Invariancia Local de Lorentz}

Los experimentos que justifican ILL e ILP, son aquellos que prueban la Relatividad Especial. Los experimentos, entre otros, son:

- El experimento clásico de Michelson-Morley y sus colaboradores [18, 19, 20, 21].

- Las pruebas de Ives-Stillwell, Rossi-Hall y otras pruebas de la dilatación del tiempo [22, 23, 24].

- Pruebas experimentales de la independencia de la velocidad de la luz respecto a la velocidad de la fuente, usando fuentes estelares de rayos $\mathrm{X}$ binarias y piones de alta energía [25, 26].

- Pruebas experimentales de la isotropía de la velocidad de la luz [27, 28, 29].

Además de estos experimentos, está la ecuación de Dirac de la mecánica cuántica relativista y sus predicciones de antipartículas y spin, la cual tuvo un éxito impresionante en la teoría relativista de la electrodinámica cuántica [30].

Un vigoroso esfuerzo teórico y experimental se ha puesto en marcha, a escala internacional, para encontrar violaciones de la relatividad especial. La motivación detrás de esto es buscar evidencia de nueva física más allá de Einstein, por ejemplo, 
aparentes violaciones de la ILL que pueden resultar de ciertos modelos de gravedad cuántica.

La gravedad cuántica afirma que hay una escala de longitud fundamental dada por la longitud de Planck, $L_{p}=\left(\hbar G / c^{3}\right)^{1 / 2}=1.6 \times 10^{-33} \mathrm{~cm}$, pero ya que la longitud no es una cantidad invariante (debido a la contracción de Lorentz-FitzGerald), entonces podría haber una violación de la ILL en algún nivel en la gravedad cuántica. En escenarios de mundo de branas, mientras la física pueda ser localmente un invariante de Lorentz en un mundo de numerosas dimensiones, el confinamiento de las interacciones de la física normal a nuestra brana tetra dimensional podría inducir efectos productos de una violación de Lorentz. Otros modelos como en la teoría de cuerdas, la presencia de escalares adicionales, de campos vectoriales y tensoriales de gran alcance que se acoplen a la materia del modelo estándar podría inducir una violación efectiva de la simetría de Lorentz. Estas y otras ideas han motivado reconsiderar como probar la ILL con mayor precisión.

Una manera simple de interpretar algunos de estos experimentos, es el formalismo $c^{2}$, el cuál sugiere que las interacciones electromagnéticas sufren una ligera violación de la ILL a través de un cambio en la velocidad de la radiación electromagnética $c$ en relación con el límite de la velocidad de las partículas de prueba ( $c_{0}$ tomará el valor de la unidad mediante una elección adecuada de unidades), en otras palabras $c \neq 1$. Esta violación necesariamente selecciona un marco en reposo universal preferido, según parece el de la radiación cósmica de fondo, a través del cual nos estamos moviendo con una rapidez de $370 \mathrm{~km} / \mathrm{s}$ [31]. Dicha interacción electromagnética, que no es invariante de Lorentz, causaría un desplazamiento en los niveles de energía de los átomos y núcleos que dependan de la orientación del eje de cuantización del estado en relación con nuestro vector de velocidad universal, y 
sobre los numeros cuánticos del estado. La presencia o ausencia de tales desplazamientos pueden ser estudiados al medir la energía de un estado en relación con otro estado que esté o no esté afectado por la supuesta violación. Una forma es buscar un cambio en los niveles de energía de los estados que habitualmente están igualmente espaciados, tal como el desacoplamiento Zeeman $2 J+1$ de los estados bases de un núcleo de spin total $J$ en un campo magnético [4]. Otra forma es comparar los niveles de un núcleo complejo con los niveles atómicos hiperfinos de un reloj máser de hidrógeno (MASER por sus siglas en inglés "Microwave Amplification by Stimulated Emission of Radiation"). La magnitud de estas anisotropías de reloj serían proporcionales a $\delta \equiv\left|c^{-2}-1\right|$.

Los primeros experimentos diseñados para medir esta anisotropía fueron los experimentos de Hughes-Drever, desarrollados en el periodo 1959-1960, y de manera independiente por Hughes y sus colaboradores en la Universidad de Yale, y por Drever en la Universidad de Glasgow [32, 33]. Los experimentos de Hughes-Drever producen resultados extremadamente precisos, dando limites sobre el parámetro $\delta \equiv c^{-2}-1$ ver Fig. 1.3. Se han realizado mejoras experimentales usando átomos y iones atrapados por enfriamiento láser (1980) [34, 35, 36]. Esta técnica reduce el ancho de las líneas de resonancia causadas por colisiones. Los valores en el parámetro $\delta$ se muestran en la Fig. 1.3 (experimentos etiquetados como NIST, U. Washington y Harvard, respectivamente).

También incluidos por comparación está el límite correspondiente con experimentos tipo Michelson-Morley (ver [37]). En estos experimentos, desde un marco preferencial, la velocidad de la luz en los dos brazos del interferómetro es $c$, aunque se puede mostrar que usando la electrodinámica del formalismo $c^{2}$ [17], la compensación debido a la contracción de Lorentz-FitzGerald del brazo paralelo es regulado 
por la velocidad $c_{0}=1$. Los experimentos de Michelson-Morley también miden el coeficiente $c^{-2}-1$. Un experimento posterior a este es el de Brillet-Hall [21] quienes usaron un interferómetro láser tipo Fabry-Perot. En experimentos recientes la frecuencia de osciladores en cavidades electromagnéticas en varias orientaciones fueron comparadas una respecto a otra, o con relojes atómicos como una función de la orientación del laboratorio [38, 39, 40, 41, 42]. Los límites de $\delta=c^{-2}-1$ están en una parte en $10^{9}$. Haugan y Lämmerzahl [43] han considerado que los límites de los experimentos tipo Michelson-Morley podrían colocarse sobre una electrodinámica modificada que implique la masa efectiva del fotón como un vector.

\subsubsection{Invariancia Local de la Posición}

La Invariancia Local de la Posición (ILP) se explica con experimentos del corrimiento gravitacional hacia el rojo. La primera prueba experimental fué propuesta por Einstein. A pesar de que Einstein consideró esto como una evidencia crucial de la Relatividad General, ahora nos damos cuenta de que no distingue entre Relatividad General y cualquier otra teoría métrica de la gravedad, es decir, es sólo una prueba de PEE. Un experimento del corrimiento gravitacional hacia el rojo mide la frecuencia o el cambio en la longitud de onda $Z \equiv \delta \nu / \nu=-\delta \lambda / \lambda$ entre dos frecuencias idénticas (relojes) colocados en reposo a dos alturas distintas en un campo gravitacional estático [44, 45]. Si la frecuencia de un tipo de reloj atómico es la misma cuando se mide en un marco local de Lorentz, independiente de la localización o velocidad del marco de referencia, entonces la comparación de las frecuencias de los relojes en reposo en diferentes ubicaciones se reduce a una comparación de las velocidades de dos marcos locales de Lorentz, uno en reposo con respecto a un reloj en el momento de emisión de su señal, el otro en reposo con 


\section{TESTS OF \\ LOCAL LORENTZ INVARIANCE}

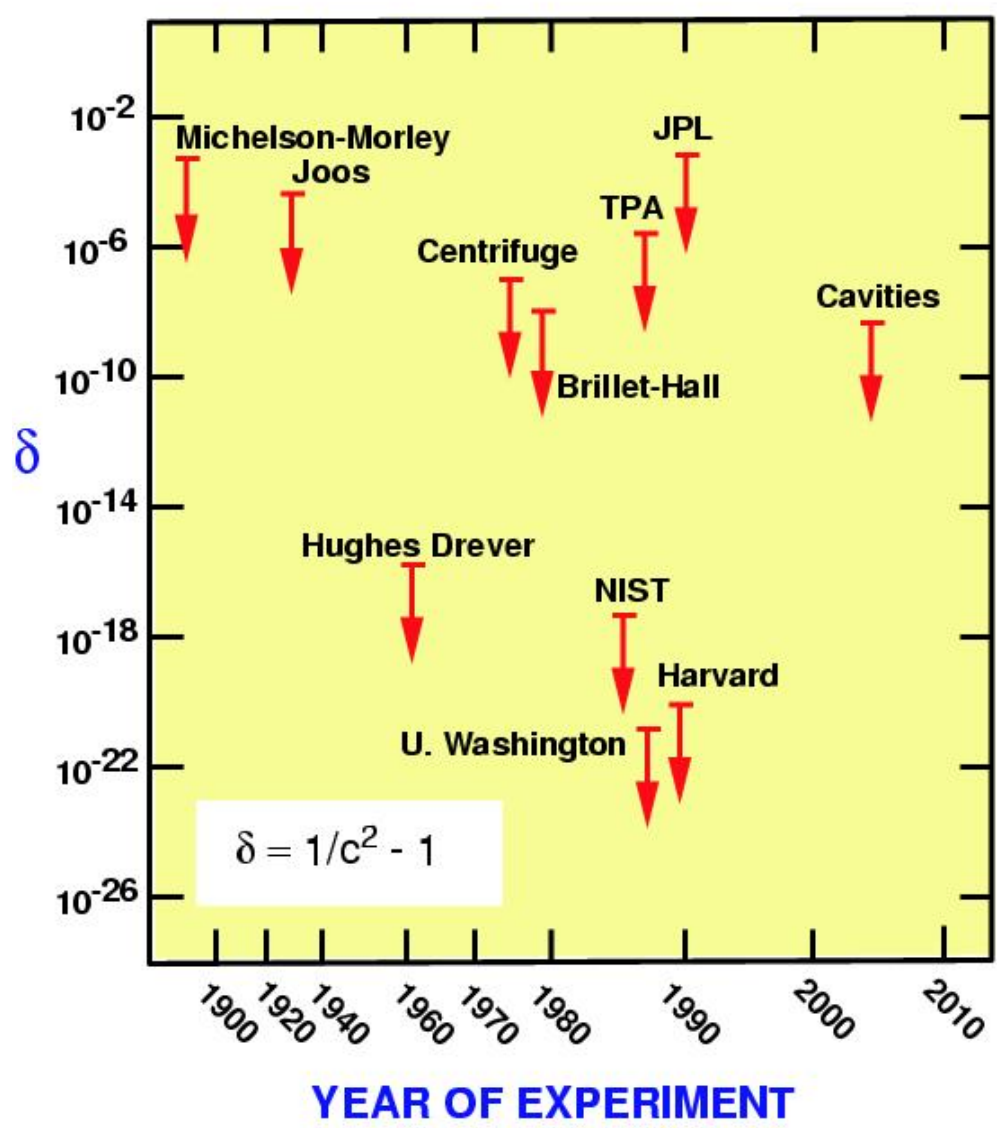

Fig. 1.3. Algunas pruebas experimentales de la ILL que muestran el límite sobre el parámetro $\delta$, el cual mide el grado de violación de la ILL en el electromagnétismo. Los experimentos de Michelson-Morley, Joos, Brillet-Hall y de cavidades prueban la isotropía de la velocidad de la luz en un viaje de ida y vuelta. Los experimentos de absorción de dos fotones (TPA por sus siglas en inglés) y el de (JPL por sus siglas en inglés) prueban la isotropía de la velocidad de la luz usando un solo camino de propagación. Los experimentos más precisos que prueban la isotropía a niveles de energía atómica son los de HughesDrever. Los límites asumen una velocidad de la Tierra de $370 \mathrm{~km} / \mathrm{s}$ con relación al marco en reposo del Universo [17. 
respecto al primer reloj en el momento de la recepción de la señal. El corrimiento en la frecuencia es entonces una consecuencia del efecto Doppler entre los marcos en cuestión. La estructura del reloj no desempeña ningún papel en absoluto. El resultado es un cambio

$$
Z=\frac{\Delta U}{c^{2}}
$$

donde $\Delta U$ es la diferencia en el potencial gravitacional Newtoniano entre los observadores que reciben y emiten la señal [44, 45]. Si ILP no es válido, entonces, resulta que el desplazamiento puede ser escrito de la siguiente forma

$$
Z=\frac{(1+\alpha) \Delta U}{c^{2}}
$$

donde el parámetro $\alpha$ puede depender del tipo de reloj (véase [1] para más detalle).

La primera medición exitosa del corrimiento al rojo fue realizada con los experimentos de Pound-Rebka-Snider 1960-1965 [44, 45], en los cuales se midió dicho parámetro por fotones de rayos gama del ${ }^{57} \mathrm{Fe}$. La precisión aumentó al hacer uso del efecto Mössbauer para producir una línea de resonancia estrecha cuyo corrimiento puede ser determinado con precisión. Otros experimentos midieron el corrimiento de las líneas espectrales en el campo gravitacional del Sol y el cambio en los relojes atómicos transportados en aviones, cohetes y satélites. En la Fig. 1.4 se resumen los experimentos más importantes del corrimiento al rojo que han sido desarrollados desde 1960. Para una revisión más detallada de estas pruebas véase [17].

PEE es la base teórica de todas las teorías métricas de la gravedad, incluyendo la Relatividad General. PEE nos ayuda a dividir las teorías gravitacionales en dos clases distintas: en teorías métricas, que son aquellas que involucran al PEE, y 


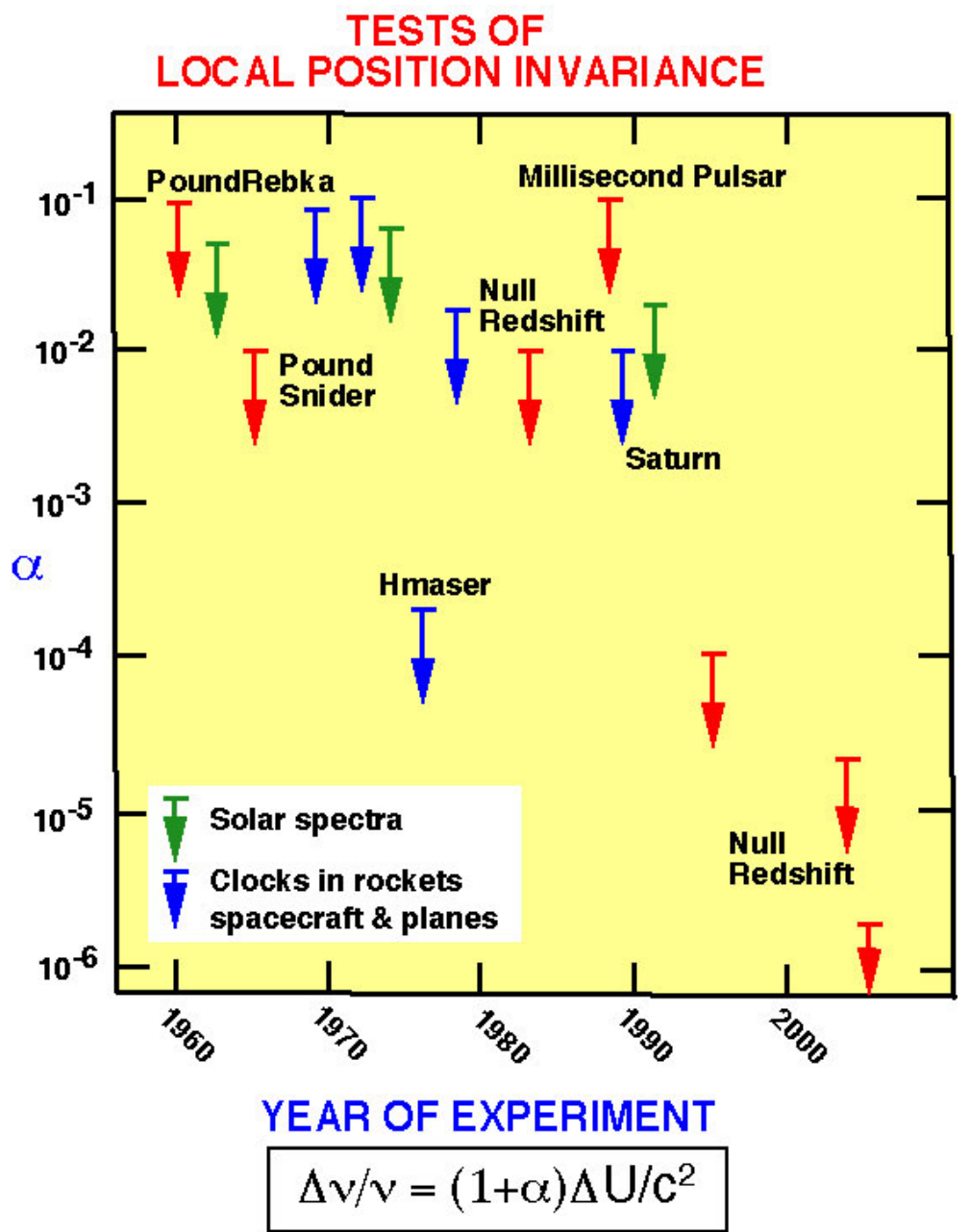

Fig. 1.4. Algunas pruebas experimentales de la ILP mediante experimentos del corrimiento al rojo gravitacional, mostrando el límite sobre el parámetro $\alpha$, el cual mide el grado de desviación del corrimiento al rojo de la formula $\Delta \nu / \nu=\Delta U / c^{2}$. En experimentos nulos del corrimiento al rojo, el límite es sobre la diferencia de $\alpha$ entre diferentes tipos de relojes [17]. 
teorías no métricas de la gravedad, aquellas teorías que no cumplen con el PEE. PEE establece [17]:

\section{La validez del PED.}

2. El resultado de cualquier prueba experimental local no-gravitacional es independiente de la velocidad del aparato en caída libre (ILL).

3. El resultado de cualquier prueba experimental local no-gravitacional es independiente de donde y cuando en el universo sea realizado el experimento (ILP).

PEE se puede resumir de la siguiente manera: cualquier experimento no-gravitacional en caída libre en presencia de un campo gravitacional es igual a un experimento no-gravitacional en ausencia de campo gravitacional [1].

Todas estas nociones dependen esencialmente de partículas puntuales y trayectorias. La Relatividad General es aplicable al movimiento de satélites, planetas y rayos de luz. Sin embargo, ya que la materia es cuántica posee muchos grados de libertad y está siempre extendida sobre una cierta región del espacio-tiempo. Una teoría de la gravedad a nivel cuántico puede ser diferente a la Relatividad General a nivel clásico y debería basarse en principio usando solo mecánica cuántica. Por ejemplo, la gravedad a nivel cuántico tiene que estar descrita por más campos que el campo de la métrica. Para explicar pruebas experimentales de los fundamentos de la relatividad general a nivel cuántico, uno debería tomar en cuenta que pueden haber más posibilidades de romper la Invariancia Local de Lorentz y la Invariancia Local de Posición a nivel cuántico que a nivel clásico. Esto no quiere decir que estemos cuantizando la gravedad, solo estamos poniendo a prueba el PEE desde el punto de vista cuántico. 


\subsection{Mecánica Estadística}

Para probar las teorías métricas de la gravitación, observaremos como la gravedad influye en los parámetros representativos de sistemas termodinámicos como la presión, número de partículas promedio, la temperatura, etc. Como una propuesta experimental estudiaremos como se ve modificada la temperatura de condensación de un gas de Bose-Einstein en presencia de un campo gravitacional homogéneo. Este resultado nos permitirá probar PEE desde un punto de vista meramente cuántico, pues el condensado de Bose-Einstein es una propiedad que está relacionada con la estadística bosónica, es decir, es una característica cuántica. PEE establece que en un marco coordenado en caída libre la temperatura de condensación deberá ser la misma temperatura que aparece en la mayoría de la literatura enfocada a estudiar los condensados de Bose-Einstein en ausencia de campos gravitacionales [46, 47, 48], por mencionar algunos.

Además el estudio de un gas bosónico confinado dentro de un recipiente de altura

finita en presencia de un campo gravitacional homogéneo es un problema actual, ya que mientras algunos autores consideran que para que se presente el fenómeno de condensación debería haber una impureza en la base del contenedor [49], en contraposición a la gran mayoría de trabajos [46, 50], y nosotros. En este trabajo se mostrará que al parecer esta impureza no es necesaria. También, se mostrará la temperatura de condensación donde el gas no se encuentra encerrado en un recipiente, más bien, se encuentra confinado por medio de osciladores anisotrópicos. En un modelo más realista, el gas bosónico es atrapado por campos electromagnéticos, por lo tanto, el volumen físico del condensado no es generado por un recipiente de 
altura finita, si no por los potenciales de confinamiento. Brevemente describiré los esquemas que han sido desarrollados para confinar átomos neutros:

- Trampas Láser.

El efecto que ha sido principalmente explotado en las trampas láser de átomos en el régimen del Condensado de Bose Einstein es el efecto dipolar, el cual se basa en la interacción del láser con el momento dipolar eléctrico inducido sobre el átomo. Por el momento ignoraremos la estructura fina e hiperfina de los átomos y definamos la frecuencia de transición de $n s \rightarrow n p$ de la siguiente forma:

$$
\Delta \equiv \hbar w_{\text {las }}-\left(E_{n p}-E_{n s}\right)=\hbar w_{\text {las }}-2 \pi \hbar c / \lambda
$$

donde $\lambda$ es la longitud de onda, $\hbar$ es la constante de Planck dividida por $2 \pi$, $w_{\text {las }}$ es la frecuencia del láser y $c$ es la velocidad de la luz.

Conviene definir la intensidad de saturación $I_{0}$, esto es, la intensidad del haz láser, la cual, cuando se encuentra en resonancia, induce una población del estado excitado (p). Dentro de un factor numérico unitario el cual depende de la polarización, $I_{0}$ está dada por:

$$
I_{0}=\frac{\epsilon_{0} c \Gamma^{2}}{d^{2}}
$$

donde $d$ es un elemento de la matriz dipolar definida apropiadamente por el elemento en cuestión, $\epsilon_{0}$ es la constante dieléctrica en el vacío (o permitividad eléctrica del vacío) y $\Gamma=\hbar / \tau$ (donde $\tau$ es la vida media del nivel de energía superior); un valor típico de $I_{0}$ es del orden de $100 \mathrm{~W} / \mathrm{m}_{2}$ [51]. Una expresión para el cambio en la energía del átomo en el campo láser es, en el límite $\Gamma \ll \Delta$,

$$
\Delta E_{\text {laser }}(\mathbf{r})=\left(\frac{I(\mathbf{r})}{I_{0}}\right) \frac{\Gamma^{2}}{\Delta}
$$


Note que en esta formula $I / I_{0}$ puede ser mayor que 1 . Una región de mayor intensidad láser proporciona un potencial atractivo para $\Delta<0$ y un potencial repulsivo para $\Delta>0$. Hay que tener en mente que para tener un arreglo de dos rayos láser que se encuentren propagándose en direcciones opuestas, el potencial debe ser variado en un escala muy corta de longitud de onda del láser, es decir, $\sim 3000 A^{\circ}$ [51].

Una pregunta importante es el grado para el cual, en todo caso, potenciales generados por los láseres son sensibles a la estructura hiperfina de Zeeman. En la medida que el acoplamiento spin-orbita en el estado excitado sea despreciable, no puede haber ningún efecto, ya que la orbita del estado base es única (estado s). Además, para un rayo láser polarizado linealmente los dos estados electrónspin son equivalentes a la inversión temporal, así cualquier efecto tendría que ser a lo mucho del orden de la proporción de la división de la estructura hiperfina de Zeeman del tunelaje, en muchos experimentos es $\leq 10^{-4}$ [52]. En un rayo láser polarizado circularmente es más complicado, por estar bastante cerca de la resonancia (separa la estructura fina).

Un factor importante en las trampas láser es que uno siempre desea, en la medida que esto sea posible, evitar el proceso de emisión espontanea. Para $\Gamma \ll \Delta$ la probabilidad que haya emisión por átomo es del orden de $\frac{I(\mathbf{r})}{I_{0}} \frac{\hbar^{-1} \Gamma^{3}}{\Lambda^{2}}$. En vista de que $\Delta^{-2}$ decae más rápido que $\Delta^{-1}$ en la ecuación $(1.16)$, conviene ajustar los láseres muy lejos de la zona de resonancia. Es posible de esta manera proporcionar potenciales generados por láser, que son de un orden mayor a la energía térmica despreciando los efectos de calentamiento del láser. Para una revisión más extensa sobre confinamiento láser ver [53]. 
- Trampas Magnéticas.

El análogo magnético del teorema de Earnshaw prohíbe que la magnitud del campo magnético B(r) tenga un máximo local en el espacio libre. Sin embargo, nada prohíbe la presencia de un mínimo local, y hay varios métodos que pueden ser usados para generar tal mínimo, los más usados son variantes de aquellos que hacen uso de un campo magnético rotante. Esta es una trampa magnética cuyo tiempo promedio, del potencial rotando ("TOP" Time-Orbiting Potential) suple los confinamientos armónicos de átomos [54], y trampas Ioffe-Pritchard [55]. Todas las trampas magnéticas puras (es decir, que no usan láser) empleadas en experimentos de condensados de Bose-Einstein hasta el momento han tenido la simetría axial y un campo de compensación finito, por ejemplo, con una elección adecuada del sistema de coordenada cilíndricas polares, la magnitud del campo toma la siguiente la forma [51]

$$
|\mathbf{B}(\mathbf{r})|=B_{0}+\frac{1}{2} \alpha \rho^{2}+\frac{1}{2} \beta z^{2}
$$

donde $\alpha$ y $\beta$ son constantes, $\rho$ y $z$ pertenecen a las coordenadas cilíndricas polares. La razón para no especificar la dirección del campo como una función de $\mathbf{r}$, aún cuando esta dirección cambie considerablemente de su valor en el origen, es que los átomos se mueven lentamente. Los átomos permanecen en el mismo estado cuántico relativo a la dirección instantánea del campo magnético; se dice que sigue las variaciones del campo magnético adiabáticamente. Así, si consideramos una especie de estructura hiperfina-Zeeman, su energía potencial será solo una función de la magnitud local del campo de la ecuación (1.16); en realidad el caso más simple será proporcional a esta energía potencial con una constante de proporcionalidad ( para más información ver [54]). 


\subsection{Desarrollo de la Tesis}

En el Capítulo dos se realizará una revisión de los conceptos fundamentales que están detrás de las teorías métricas de la gravedad, en especial el Principio de Equivalencia de Einstein. En el Capítulo tres recordaremos algunos conceptos de Mecánica Estadística, en particular, lo relacionado con la condensación de BoseEinstein en ausencia de campos gravitacionales, así como una revisión más a fondo de los potenciales de confinamiento. El Capítulo cuatro es la parte central del trabajo. En el mostraremos los resultados obtenidos al considerar un gas de BoseEinstein en presencia de un campo gravitacional homogéneo. Nos enfocaremos en estudiar las modificaciones de las propiedades termodinámicas de dicho gas, como la presión, el número de partículas y la temperatura de condensación. Esto último lo haremos de dos formas. Primero consideraremos un gas de Bose-Einstein encerrado en un contenedor de altura finita, después consideraremos que el gas de Bose-Einstein se encuentra confinado por osciladores anisotrópicos. Por ultimo daremos nuestras conclusiones y perspectivas respecto a la propuesta experimental que pone a prueba el Principio de Equivalencia de Einstein. 
CAPÍtulo 2

\section{Fundamentos de la teoría de la relatividad general}

En 1915 Einstein propuso una modificación a la descripción del espacio-tiempo de sistemas físicos de la Relatividad Especial. En está descripción, como en la más antigua de Newton, la geometría del espacio-tiempo fué fijada de una vez por todas; esta no será afectada por la presencia de otros sistemas físicos existentes en el espacio tiempo.

Antes de continuar mencionaremos una propiedad importante del conjunto de todos los mapeos permitidos de la variedad del espacio-tiempo dentro de si mismos es que ellos forman un grupo. Los requisitos para formar un grupo se cumplen en el caso de los mapeos en la variedad, si tomamos el producto de dos mapeos ser el resultado de realizar primero un mapeo y después el otro. Ya que el resultado de realizar dos mapeos en sucesión depende del orden en que sean realizados, el grupo de mapeos no es conmutativo. La topología de la variedad limita los mapeos permitidos. Este grupo también dependerá de la topología y pueden ser usados en parte para caracterizar esta topología. De aquí en adelante este grupo será referido como el grupo mapeado en la variedad. En nuestra terminología, la 
geometría aparecerá como un elemento absoluto en todas las teorías cuyo grupo covariante es el grupo mapeado en la variedad. El propósito de Einstein consistió en remover la geometría del espacio-tiempo del reino de lo absoluto al considerar que un elemento dinámico debe de estar en igualdad de condiciones con cualquier otro elemento dinámico del sistema físico. Este marco es conocido hoy en día como la Relatividad General [56].

La geometría del espacio-tiempo en la Relatividad General está caracterizada por una métrica Riemanniana, como en la Relatividad Especial [56]. Siendo un elemento dinámico estará determinada por un conjunto de leyes dinámicas de igual forma que el campo electromagnético es determinado por las ecuaciones de Maxwell-Lorentz.

Einstein fué guiado a buscar un marco más general que el proporcionado por la Relatividad Especial. En particular el estaba preocupado por la inhabilidad de la mecánica Newtoniana o de la Relatividad Especial para explicar la constante universal de la proporción de la masa inercial y masa gravitacional pasiva de los cuerpos materiales. Además, se opuso a la existencia de ciertos tipos de movimientos absolutos, es decir, movimientos acelerados en estas dos teorías. Einstein creía que todo movimiento debería ser relativo, no solo movimiento uniforme.

\subsection{El Principio de Equivalencia Débil}

Einstein consideró que la geometría del espacio-tiempo debería ser un elemento dinámico en todas las teorías físicas. Su principio de equivalencia sugiere que la geometría y la gravitación son la misma cosa. PED fué una consecuencia del reconocimiento de Einstein de la importancia de un hecho experimental que, hasta 
entonces, había sido considerado ser un accidente de la naturaleza. Este accidente es la aparente constancia de la razón entre masa inercial y masa gravitacional pasiva, $m_{i} / m_{p}=\alpha$, para cuerpos materiales.

Galileo observó que en el campo gravitacional de la tierra todos los cuerpos caen con la misma aceleración. Esto no es cierto para el movimiento de cuerpos cargados en un campo eléctrico. La observación de Galileo dio indicios de una propiedad muy especial del campo gravitacional. En la teoría gravitacional de Newton y en la teoría de la Relatividad Especial se asumió como una hipótesis adicional la constancia de $\alpha$. Esto debería ser una consecuencia de estas teorías. Esta premisa ha sido sometido a muchas pruebas experimentales, desde Galileo hasta sofisticados experimentos realizados hoy en día. Podemos tomar esto como un hecho experimental muy bien establecido que la razón de $\alpha$ para cuerpos materiales de todos los tipos es una constante universal. Si $\alpha$ tiene el mismo valor para todos los objetos masivos, todos ellos deberían comportarse de la misma forma. Para partículas moviéndose en un campo gravitacional recordemos que las ecuaciones de movimiento están dadas de la siguiente forma [56]:

$$
\frac{d^{2} z^{\mu}}{d \lambda^{2}}+\Gamma_{\rho \sigma}^{\mu} \frac{d z^{\rho}}{d \lambda} \frac{z^{\sigma}}{d \lambda}=0
$$

para una adecuada elección del parámetro $\lambda$. Las observaciones sobre el movimiento de todas las partículas libres servirían para determinar la misma afinidad (Las cantidades $\Gamma_{\rho \sigma}^{\mu}$ que son las componentes de un objeto geométrico definido sobre la variedad las llamaremos conexión afín o afinidad [56]) en una región del espaciotiempo dado. Einstein continuó con la generalización de sus resultados para todos los sistemas físicos, el cual llamamos aquí PED. PED se puede enunciar como ya se dijo antes de la siguiente forma: si un cuerpo de prueba sin carga eléctrica es puesto en un evento inicial en el espacio-tiempo y después se le da una velocidad 
inicial, entonces su trayectoria subsecuente será independiente de su estructura interna y composición [1]. Aquí las señales de luz en un campo gravitacional débil deberían propagarse a lo largo de rayos determinados por la misma afinidad como aparece en las ecuaciones de movimiento de partículas 2.1. En realidad no es obvio que PED sea universalmente válido, no obstante, a pesar de los pocos datos que tenemos parece estar bien sustentado. Deberíamos hablar de la posibilidad que discutió Trautman [57] de que la actual afinidad física sea asimétrica, en cuyos casos tendrían que existir sistemas físicos que pudieran ser usados para determinar está parte antisimétrica. (De hecho, si tales sistemas no existen en la naturaleza, no hay que considerar una afinidad asimétrica.) Ya que las mediciones efectuadas en el movimiento de partículas y rayos de luz (haces de luz) pueden servir para determinar solo la parte simétrica de la afinidad, PED tendría que generalizarse en este caso.

PED propone, en un sentido aproximado, que sería significativo considerar la afinidad como si fuera plana en una región donde el campo gravitacional sea débil. Esto también sugiere que deberíamos tratar la aparición de la afinidad en las ecuaciones de movimiento de un sistema físico como dinámicos, más que un absoluto, ya que esta es una manifestación del campo gravitacional. PED es sólo aplicable en aquellos casos donde uno pueda despreciar la acción de un sistema físico sobre las fuentes de cualquier campo gravitacional que puedan estar presentes. Por lo tanto, si usáramos un cuerpo suficientemente masivo para medir la afinidad en alguna región del espacio-tiempo, está podría interactuar fuertemente con dichas fuentes, y por lo tanto la afinidad determinada por observaciones del movimiento de partículas podría ser diferente a la determinada al considerar un cuerpo menos masivo. Podría ser que utilizando un número diferente de sistemas físicos se pudiera 
separar, en una única manera, una afinidad plana de entre las varias afinidades determinadas por las observaciones hechas sobre los sistemas. También existe la posibilidad, de que por medio de las observaciones hechas en sistemas llevados lo suficientemente lejos de las fuentes de campo gravitacional se pueda separar solo una variedad plana de cualquier afinidad que este presente en cualquier región del espacio-tiempo dado.

El argumento que Einstein utilizó para el PED es su famoso experimento pensado del elevador en caída libre. El imaginó un elevador que estaba cayendo libremente en un campo gravitacional. Para un observador dentro del elevador, un cuerpo material que estuviera también cayendo libremente parecería estar en un estado de movimiento uniforme. De la misma forma, en un elevador acelerado en una región del espacio en ausencia de gravedad, un cuerpo material parecería comportarse como si estuviera cayendo en un campo gravitacional uniforme. También, los rayos de luz podrían aparentar viajar a lo largo de trayectorias curvas en su ultima descripción del elevador. Esto fué, en realidad, el argumento que Einstein dio para concluir que la trayectoria de un rayo de luz debería ser afectado por un campo gravitacional. Con este experimento pensado Einstein concluyó que debería ser imposible, sobre la base de mediciones puramente locales, distinguir entre el movimiento uniforme de un observador en un campo gravitacional y el movimiento acelerado en un espacio libre de gravedad. El fué guiado por la noción de relatividad de todo movimiento. Esta fue la razón para nombrar a este nuevo marco como la teoría de la Relatividad General [56].

Varios autores han tomado la equivalencia local de la gravitación y los efectos de la aceleración como la base para el desarrollo de la Relatividad General y lo han llamado PED. Hoy en día esto constituye una suposición separada so- 
bre y acerca del PED. Este postulado que sólo determina la afinidad de que el campo gravitacional aparezca en las leyes dinámicas de estos sistemas, hasta el punto de que estas leyes sean leyes locales. Estos nuevos planteamientos obedecen el hecho de que las mediciones realizadas en un marco de referencia acelerado en un espacio plano pueden ser descritas al realizar un mapeo tal que la afinidad del espacio-tiempo plano ya no tiene sus valores en Minkowski $\left(\eta_{\mu \nu}\right)$. Alternativamente, uno puede siempre encontrar un mapeo, esto es un marco de referencia, tal que cualquier afinidad pueda estar descrita al tomar los valores de Minkowski y hacer sus derivadas nulas en punto de la variedad del espacio tiempo. Observamos que asumir equivalencia local entre efectos debidos a la aceleración y efectos gravitacionales requieren una región suficientemente pequeña del espacio-tiempo, donde las leyes de la Relatividad Especial sean válidas.

Como esto es una suposición adicional, debemos de aquí en adelante, referirnos a la equivalencia local de los efectos gravitación y los efectos inerciales como el principio de mínimo acoplamiento del campo gravitacional a la materia. La principal dificultad en aplicar estos principios yace en el uso de la palabra "local" que significa una región suficientemente pequeña en el espacio-tiempo. Aquí podemos imaginar una partícula sin dimensiones que está caracterizada por una masa m, además de, un tensor de spin $\sigma^{\mu \nu}$, por ejemplo, un electrón. Sus trayectorias podrían entonces estar determinadas por la ecuación de la geodésica, a la cual se le podría agregar un termino proporcional a $R_{\mu \nu \tau \kappa} \sigma^{\mu \nu} \sigma^{\tau \kappa}$. Una ley dinámica podría violar el principio de mínimo acoplamiento como se ha definido. Sin embargo, también se puede argumentar que una partícula, tal como la hemos considerado aquí, no es en realidad una estructura local y por lo tanto no viola el principio, pero esta objeción es una sutileza; si tal partícula realmente existiera en la naturaleza, uno 
nunca podría encontrar una región del espacio-tiempo lo suficientemente pequeño para causar que el término de spin desaparezca.

Es importante reconocer las diferencia entre PED y mínimo acoplamiento. El primero no es local y es crucial para el desarrollo del PEE . El principio de mínimo acoplamiento puede o no puede tener validez universal. Esta violación de ninguna forma afecta la validez de las teorías métricas de la gravedad, al menos que uno insistiera en tomarlo como si fuera un principio de esta teoría. Los efectos causados por la aceleración no son iguales a lo efectos geométricos. Una partícula libre en un espacio plano se mueve a lo largo de una geodésica de la métrica plana, esto es un hecho geométrico. Que parezca que se mueva a lo largo de una trayectoria curva cuando se observa desde un marco de referencia acelerado no cambia este hecho. Los efectos geométricos son inherentes a los sistemas físicos considerados mientras los efectos debidos a la aceleración son extrínsicos a los sistemas físicos considerados.

\subsection{Principio de Equivalencia de Einstein}

Antes de enunciar PEE es útil definir un experimento local no-gravitacional como cualquier experimento (i) realizado en un laboratorio en caída libre protegido y lo suficientemente pequeño para que las inhomogeneidades de los campos externos puedan ser despreciadas en todo su volumen, y (ii) en el cual los efectos autogravitacionales sean ignorados. Por ejemplo, una medición de la constante de estructura fina es una prueba experimental local no-gravitacional.

El Principio de Equivalencia de Einstein establece:

1) La validez del PED. 
2) El resultado de cualquier prueba experimental local no-gravitacional será independiente de la velocidad del aparato en caída libre (ILL).

3) El resultado de cualquier prueba experimental no-gravitacional será independiente de donde y cuando en el universo el experimento sea realizado (ILP).

Este principio es la base de las teorías de la gravedad, por eso es posible argumentar que si PEE es válido, entonces la gravitación define un espacio-tiempo curvo, es decir, debe satisfacer los postulados de las Teorías Métricas de la Gravedad. Estos postulados establecen:

a) El espacio-tiempo esta dotado con una métrica $\mathbf{g}$.

b) Las líneas de mundo de los cuerpos de prueba son geodésicas de esta métrica.

c) En un marco local cayendo libremente, llamados marcos locales de Lorentz, las leyes de la física no-gravitacional son aquellas de la Relatividad Especial.

Relatividad General, la teoría de Brans-Dicke [58], y la teoría bimétrica de Rosen [59] son teorías métricas de la gravedad. Sin embargo, la teoría de BelinfanteSwihart [60, 61, 62] no es una teoría métrica de la gravedad.

El argumento es el siguiente. La validez de PED dota a un espacio-tiempo con una familia de trayectorias preferidas, las líneas de mundo de partículas de prueba cayendo libremente. En un marco local que sigue una de estas trayectorias, las partículas de prueba se mueven en una línea recta a velocidades constantes. Además, los resultados de pruebas experimentales locales no-gravitacionales son independientes de la velocidad del marco. En dos de estos marcos localizados en el mismo evento, $\mathfrak{P}$, en el espacio-tiempo pero moviéndose relativamente un marco respecto al otro, todas las leyes de la Física no-gravitacionales deben de hacer las mismas predicciones para experimentos idénticos, es decir, deberán ser invariantes 
de Lorentz. A este aspecto de PEE se le conoce como la Invariancia Local de Lorentz (ILL). Por lo tanto, deben existir en el universo uno o más campos tensoriales de segundo rango $\psi^{(1)}, \psi^{(2)}, \ldots$, que se reducen en un marco local en caída libre a los campos que son proporcionales a la métrica de Minkowski, $\phi^{(1)}(\mathfrak{P}) \eta, \phi^{(2)}(\mathfrak{P}) \eta, \ldots$, donde $\phi^{(A)}(\mathfrak{P})$ son campos escalares que pueden variar de evento a evento. Diferentes miembros de este conjunto de campos pueden acoplarse a diferentes campos no-gravitacionales, tales como campos bosónicos, campos fermiónicos y campos electromagnéticos, etc. Por lo tanto, el resultado de una prueba experimental local no-gravitacional debe también ser independiente de la localización del espaciotiempo del marco. A esto se le conoce como la Invariancia Local de la Posición (ILP).

Hay dos posibilidades:

i) Las versiones locales de $\psi^{A}$ deben tener coeficientes constantes, es decir, los campos escalares $\phi^{(A)}(\mathfrak{P})$ deben de ser constantes. Esto es posible por un simple rescalamiento de coordenadas y el acoplamiento de las constantes (semejante a la unidad de carga eléctrica) para cada conjunto de campos escalares iguales a la unidad en cualquier marco local.

ii) Los campos escalares $\phi^{(A)}(\mathfrak{P})$ deberán ser múltiplos constantes de un solo campo escalar $\phi^{()}(\mathfrak{P})$, es decir, $\phi^{(A)}(\mathfrak{P})=c^{A} \phi^{()}(\mathfrak{P})$. Si esto es cierto, entonces cantidades medibles físicamente, serán adimensionales, serían independientes de la posición (esencialmente, el campo escalar se anulará). Un ejemplo es una medición de la constante de estructura fina; otro ejemplo es una medición de la longitud de una varilla rígida en centímetros, ya que tal medición es una proporción entre la longitud de la varilla y el de una varilla estándar cuya longitud es de un centímetro. Así, una combinación de un rescalamiento de constantes de 
acoplamiento para poner las $c^{A}$ iguales a la unidad (redefinición de unidades), junto con una transformación conforme para un nuevo campo $\bar{\psi}=\phi^{-1} \psi$, garantiza que la versión local de $\bar{\psi}$ será $\eta$.

En cualquiera de los dos casos, concluimos que existen campos que se reducen a $\eta$ en cualquier marco local en caída libre. La geometría diferencial básica 63 nos muestra que estos campos son uno solo y el mismo: un único campo tensorial de segundo rango simétrico que denotaremos como g. Esta g tiene la propiedad de que posee una familia de líneas de mundo llamadas geodésica, y que en cada evento $\mathfrak{P}$ existen marcos locales, llamados marcos locales de Lorentz, que siguen estas geodésica, en el cual

$$
g_{\mu \nu}(\mathfrak{P})=\eta_{\nu \mu}+0\left(\sum_{\alpha}\left|x^{\alpha}-x^{\alpha}(\mathfrak{P})\right|^{2}\right), \quad \partial g_{\mu \nu} / \partial x^{\alpha}=0, \quad \text { en } \mathfrak{P}
$$

Por lo tanto, las geodésicas son líneas rectas en marcos locales de Lorentz, como son las trayectorias de cuerpos de pruebas en marcos locales que se encuentren en caída libre, de ahí que los cuerpos de prueba se muevan sobre geodésicas de g y los marcos locales de Lorentz coincidan con los marcos en caída libre. 
Capítulo 3

\section{Condensación de Bose Einstein en ausencia de campos gravitacionales y fuerzas externas}

En esta sección describiré brevemente la termodinámica de una gas ideal de Bose-Einstein (BE). Las partículas que obedecen a la estadística de Bose Einstein tienen un comportamiento muy distinto a las partículas que obedecen la estadística de Fermi-Dirac (FD). Debido a que la función de onda es simétrica, no implica la existencia de un principio de exclusión de manera que en cada estado propio pueden coexistir cuantas partículas se quieran [64]. Si la temperatura tiende a cero las partículas se distribuirán en los estados de menor energía, así que podríamos sospechar que cerca del cero absoluto en la temperatura las partículas ocuparan el estado de menor energía, en este caso es $\varepsilon=0$. Para estudiar constitutivamente como se lleva a cabo esta distribución de partículas a diferentes temperaturas recordemos que la distribución de BE para partículas indistinguibles que no interactúan entre si esta dada por la siguiente ecuación [64].

$$
n_{i}^{*}=\frac{g_{i}}{e^{\beta\left(\varepsilon_{i}-\mu\right)}-1} .
$$


En la ecuación (3.1), $g_{i}$ es el número de subniveles de energía por cada nivel de energía $\varepsilon_{i}, \beta=\frac{1}{\kappa T}, \mu$ es el potencial químico del gas y $\kappa$ es la constante de Boltzmann. Contrario al caso de Fermi-Dirac, aquí $\mu$ nunca puede ser un número positivo, pues si lo fuera habría valores $\varepsilon_{i}$ para los cuales $\varepsilon_{i}-\mu$ daría lugar a valores negativos de $n_{i}^{*}$ lo cual no tiene sentido. Para el caso de BE y partículas encerradas en una caja $-\infty \leq \mu \leq 0$; el máximo valor que pueda tomar $\mu$ es cero. Otra propiedad singular de la ecuación (3.1) es la ocupación del estado base a bajas temperaturas. Si llamamos $n_{0}^{*}$ al número de partículas en $\varepsilon_{0}=0$ tenemos que,

$$
n_{0}^{*}=\frac{g_{0}}{e^{-\beta \mu}-1}
$$

donde $g_{0}$ es la degeneración del estado base, ya que $\mu$ es finito y negativo cuando $\mu \rightarrow 0, e^{-\beta \mu} \rightarrow 1$ para $\beta$ fija $n_{0}^{*}$ crece sin límite, esto es, la población del estado base podría pensarse que sería tal que $n_{0}^{*} \simeq N$ donde $N$ es el número total de partículas en el sistema. Para entender mejor este efecto recordemos algunas resultados entre la conexión de la mecánica estadística y la termodinámica. En el ensemble gran canónico tenemos que la presión esta dada por:

$$
\frac{P V}{\kappa T} \equiv \ln \Xi=-\sum_{\varepsilon} \ln \left(1-z e^{-\beta \varepsilon}\right),
$$

donde $\Xi$ es la función de partición gran canónica, y el número total de partícula se define como:

$$
N \equiv \sum_{\varepsilon}\left\langle n_{\varepsilon}\right\rangle=\sum_{\varepsilon} \frac{1}{z^{-1} e^{\beta \varepsilon}-1},
$$

donde $\beta=1 / \kappa T$, mientras que z es la fugacidad del gas la cual está relacionado con el potencial químico mediante la siguiente definición:

$$
z \equiv e^{\mu / \kappa T}
$$


Como podemos notar $z e^{-\beta \varepsilon}$, para toda $\varepsilon$, es menor que la unidad. Para grandes volúmenes de $\mathrm{V}$, el espectro de energía de partícula individual bajo está aproximación se puede considerar como un continuo. Las sumas de las ecuaciones (3.3) y (3.4) pueden ser reemplazadas por una integral. Para hacer esto, usaremos la expresión asintótica para la densidad de estados $a(\varepsilon)$ en la vecindad de una energía dada $\varepsilon$, es decir:

$$
a(\varepsilon) d \varepsilon=\left(2 \pi V / h^{3}\right)(2 m)^{3 / 2} \varepsilon^{1 / 2} d \varepsilon
$$

Hay que tener mucho cuidado al sustituir la densidad de estados cuando se cambia la suma por una integral, hay que tomar el nivel de energía $\varepsilon=0$ fuera de la suma en cuestión antes de llevar a cabo la integración

$$
\frac{P}{\kappa T}=-\frac{2 \pi}{h^{3}}(2 m)^{3 / 2} \int_{0}^{\infty} \varepsilon^{1 / 2} \ln \left(1-z e^{-\beta \varepsilon}\right) d \varepsilon-\frac{1}{V} \ln (1-z),
$$

y

$$
\frac{N}{V}=\frac{2 \pi}{h^{3}}(2 m)^{3 / 2} \int_{0}^{\infty} \frac{\varepsilon^{1 / 2} d \varepsilon}{z^{-1} e^{\beta \varepsilon}-1}+\frac{1}{V} \frac{z}{1-z},
$$

el límite más bajo de la integral puede ser tomado como 0 , pues el estado $\varepsilon=0$ no contribuye en la integral de ninguna forma. Antes de realizar las integrales, vale la pena realizar un comentario acerca de la importancia relativa del último término de las ecuaciones (3.7), (3.8). Para $z \ll 1$, el cual corresponde a situaciones no muy lejanas del límite clásico, cada uno de estos términos es del orden de $\frac{1}{N}$, y por lo tanto, despreciables. En todo caso, como z incrementa y asume valores cercanos a la unidad, el término $\frac{z}{(1-z) V}$ en la ecuación 3.8, el cual es igual a $\frac{N_{0}}{V}$ (donde $N_{0}$ es el número de partículas en el estado base $\varepsilon=0$ ), puede llegar a ser una fracción significante de la cantidad $\frac{N}{V}$; está acumulación de una fracción macroscópica de las partículas dadas dentro de un estado $\varepsilon=0$ conduce al fenómeno de condensación de Bose-Einstein. Sin embargo, ya que $\frac{z}{(1-z)}=N_{0}$ y por lo tanto $z=\frac{N_{0}}{\left(N_{0}+1\right)}$, 
el término $-V^{-1} \ln (1-z)$ de la ecuación (3.7) es igual $V^{-1} \ln \left(N_{0}+1\right)$, el cual es proporcional a $O\left(N^{-1} \ln N\right)$; este término es despreciable para cualquier valor de $z$ y por lo tanto puede ser desechado completamente. Ahora procedamos a resolver las integrales de las ecuaciones (3.7) y (3.8), para ello realizaremos el siguiente cambio de variable $\beta \varepsilon=\frac{p^{2}}{(2 m \kappa T)}=x$, sustituyendo para la ecuación de la presión se obtiene:

$$
\frac{P}{\kappa T}=-\frac{2 \pi(2 m \kappa T)^{3 / 2}}{h^{3}} \int_{0}^{\infty} x^{1 / 2} \ln \left(1-z e^{-x}\right) d x=\frac{1}{\Lambda^{3}} g_{5 / 2}(z),
$$

y para el número de partículas en el estado excitado tenemos

$$
\frac{N-N_{0}}{V}=\frac{2 \pi(2 m \kappa T)^{3 / 2}}{h^{3}} \int_{0}^{\infty} \frac{x^{1 / 2} d x}{z^{-1} e^{x}-1}=\frac{1}{\Lambda^{3}} g_{3 / 2}(z),
$$

donde

$$
\Lambda=\frac{h}{(2 \pi m \kappa T)^{1 / 2}},
$$

es la longitud térmica de de Broglie y $g_{\nu}(z)$ son las funciones de Bose-Einstein [46],

$$
g_{\nu}(z)=\frac{1}{\Gamma(\nu)} \int_{0}^{\infty} \frac{x^{\nu-1} d x}{z^{-1} e^{x}-1}=z+\frac{z^{2}}{2^{\nu}}+\frac{z^{3}}{3^{\nu}}+\cdots
$$

La energía interna de este sistema está dada por la siguiente ecuación

$$
\begin{aligned}
U & \equiv-\left(\frac{\partial}{\partial \beta} \ln \Xi\right)_{z, V}=\kappa T^{2}\left\{\frac{\partial}{\partial T}\left(\frac{P V}{\kappa T}\right)\right\}_{z, V}=\kappa T^{2} V g_{5 / 2}(z)\left\{\frac{d}{d T}\left(\frac{1}{\Lambda^{3}}\right)\right\} \\
& =\frac{3}{2} \kappa T \frac{V}{\Lambda^{3}} g_{5 / 2}(z),
\end{aligned}
$$

aquí, hemos usado la ecuación (3.9), además del hecho de que $\Lambda \propto T^{-1 / 2}$. Si sustituimos la ecuación (3.9) en la ecuación (3.13) obtenemos la siguiente relación

$$
P=\frac{2}{3}(U / V)
$$


cabe mencionar que el término $\frac{2}{3}$ es debido a que se ha considerado un gas de Bose-Einstein en tres dimensiones, cuyo espectro de energía está dado por $\varepsilon \propto p^{2}$, en ausencia de interacciones entre partículas y campos externos, además de no tener grados de libertad internos. En el caso más general donde se tenga un gas de Bose-Einstein de n-dimensiones cuyo espectro energía de partícula individual este dado por $\varepsilon \propto p^{s}$, en ausencia de interacciones entre los constituyentes del gas y campos externos, se obtiene

$$
P=\frac{s}{n}(U / V)
$$

Para pequeños valores de $z$, podemos hacer uso del desarrollo de la expresión (3.12), al mismo tiempo que despreciamos $N_{0}$ en comparación con $N$. Una eliminación de $z$ entre las ecuaciones (3.9) y (3.10) nos ayuda a invertir la serie de la ecuación (3.10), para obtener un desarrollo para $z$ en potencias de $n \Lambda^{3}$ y sustituir este desarrollo dentro de la serie que aparece en la ecuación (3.9). La ecuación de estado toma la forma de un desarrollo virial,

$$
\frac{P V}{N \kappa T}=\sum_{l=1}^{\infty} a_{l}\left(\frac{\Lambda^{3}}{v}\right)^{l-1}
$$

donde $v(\equiv 1 / n)$ es el volumen por partícula, los coeficientes $a_{l}$, los cuales son referidos como los coeficientes del virial del sistema, resultan ser:

$$
\begin{aligned}
& a_{1}=1 \\
& a_{2}=-\frac{1}{4 \sqrt{2}}=-0.17678 \\
& a_{3}=-\left(\frac{2}{9 \sqrt{3}}-\frac{1}{8}\right)=-0.00330 \\
& a_{4}=-\left(\frac{3}{32}+\frac{5}{32 \sqrt{2}}-\frac{1}{2 \sqrt{6}}\right)=-0.00011
\end{aligned}
$$

y así sucesivamente. Para el calor específico del gas tenemos: 


$$
\begin{aligned}
\frac{C_{V}}{N \kappa} & \equiv \frac{1}{N \kappa}\left(\frac{\partial U}{\partial T}\right)_{N, V}=\frac{3}{2}\left\{\frac{\partial}{\partial T}\left(\frac{P V}{N \kappa}\right)\right\}_{v}=\frac{3}{2} \sum_{l=1}^{\infty} \frac{5-3 l}{2} a_{l}\left(\frac{\Lambda^{3}}{v}\right)^{l-1} \\
& =\frac{3}{2}\left[1+0.0884\left(\frac{\Lambda^{3}}{v}\right)+0.0066\left(\frac{\Lambda^{3}}{v}\right)^{2}+0.0004\left(\frac{\Lambda^{3}}{v}\right)^{3}+\cdots\right]
\end{aligned}
$$

Cuando $T \rightarrow \infty$ (y por lo tanto $\Lambda \rightarrow 0$ ), la presión y el calor específico del gas se aproximan a sus valores clásicos, es decir, $n \kappa T$ y $\frac{3}{2} N \kappa$, respectivamente. A medida que la temperatura del sistema disminuye (y el valor del parámetro $\frac{\Lambda^{3}}{v}$ aumenta), los desarrollos de las ecuaciones (3.16) y (3.18) ya no son útiles. Para estos valores de la temperatura tenemos que trabajar con las ecuaciones $3.9,3.10$ y $(3.13)$. El valor de $z$ es ahora obtenido de la ecuación (3.10), el cual puede ser escrito como

$$
N_{e}=V \frac{(2 \pi m \kappa T)^{3 / 2}}{h^{3}} g_{3 / 2}(z),
$$

donde $N_{e}$ es el número de partículas en el estado excitado $(\varepsilon \neq 0)$, de hecho, a menos que $z$ este muy cerca de la unidad, $N_{e} \simeq N$. Para $0 \leq z \leq 1$, la función de Bose-Einstein $g_{3 / 2}(z)$ incrementa monótonamente con $z$ y es acotada, su máximo valor está dado por

$$
g_{3 / 2}(1)=1+\frac{1}{2^{3 / 2}}+\frac{1}{3^{3 / 2}}+\cdots \equiv \zeta\left(\frac{3}{2}\right) \simeq 2.612 .
$$

Para más detalle acerca de las funciones de Bose-Einstein ver [46]. Por lo tanto, para todo valor de $z$ que nos interesa tenemos:

$$
g_{3 / 2}(z) \leq \zeta\left(\frac{3}{2}\right)
$$

Para un volumen $V$ y una temperatura $T$ dada, el número total de partículas en todos los estados excitados están también acotados, es decir:

$$
N_{e} \leq V \frac{(2 \pi m \kappa T)^{3 / 2}}{h^{3}} \zeta\left(\frac{3}{2}\right) .
$$


Ahora, siempre y cuando el número de partículas en el sistema sea menor que este valor límite, prácticamente todas las partículas en el sistema están distribuidas sobre los estados excitados y el valor exacto de $z$ es determinado por la ecuación (3.19), con $N_{e} \simeq N$. Si el número de partículas excede este valor límite, entonces es natural que los estados excitados recibirán más partículas de las que puedan contener, es decir

$$
N_{e}=V \frac{(2 \pi m \kappa T)^{3 / 2}}{h^{3}} \zeta\left(\frac{3}{2}\right),
$$

mientras que el resto de partículas ocuparán el estado base $\varepsilon=0$ (cuya capacidad, bajo todas las circunstancias, es prácticamente ilimitado):

$$
N_{0}=N-\left\{V \frac{(2 \pi m \kappa T)^{3 / 2}}{h^{3}} \zeta\left(\frac{3}{2}\right)\right\} .
$$

El valor preciso de $z$ es ahora determinado por la siguiente formula

$$
z=\frac{N_{0}}{N_{0}+1} \simeq 1-\frac{1}{N_{0}}
$$

el cual, para todo propósito práctico, es la unidad. Este fenómeno, un gran número de partículas acumuladas en un solo estado cuántico $(\varepsilon=0)$, es llamado condensación de Bose-Einstein. En cierto sentido, este fenómeno es similar al proceso más familiar de un condensado de vapor dentro del estado líquido, el cual toma lugar en un espacio físico ordinario. Pero conceptualmente los dos procesos son muy diferentes. Primero, el fenómeno de condensación de Bose-Einstein es de origen puramente cuántico (ocurriendo aún en ausencia de fuerzas intermoleculares); Segundo, este fenómeno toma lugar en el espacio de momentos y no en el espacio de coordenadas.

La condición para que se inicie la condensación es:

$$
N>V T^{3 / 2} \frac{(2 \pi m \kappa T)^{3 / 2}}{h^{3}} \zeta\left(\frac{3}{2}\right),
$$


o, si mantenemos constante el número total de partículas $N$ y el volumen $V$ y variamos la temperatura $T$, obtenemos

$$
T<T_{c}=\frac{h^{2}}{2 \pi m \kappa}\left\{\frac{N}{V \zeta \frac{3}{2}}\right\}^{2 / 3},
$$

$T_{c}$ denota una temperatura característica que depende de la masa de las partículas $m$ y de la densidad de las partículas $\frac{N}{V}$ en el sistema. En consecuencia, el sistema puede ser observado como una mezcla de dos fases:

i) una fase normal, la cual consiste de $N_{e}=N\left(T / T_{c}\right)^{3 / 2}$ partículas distribuidas sobre los estados excitados $(\varepsilon \neq 0)$, y

ii) una fase condensada, la cual consiste de $N_{0}=\left(N-N_{e}\right)$ partículas acumuladas en el estado base $(\varepsilon=0)$.

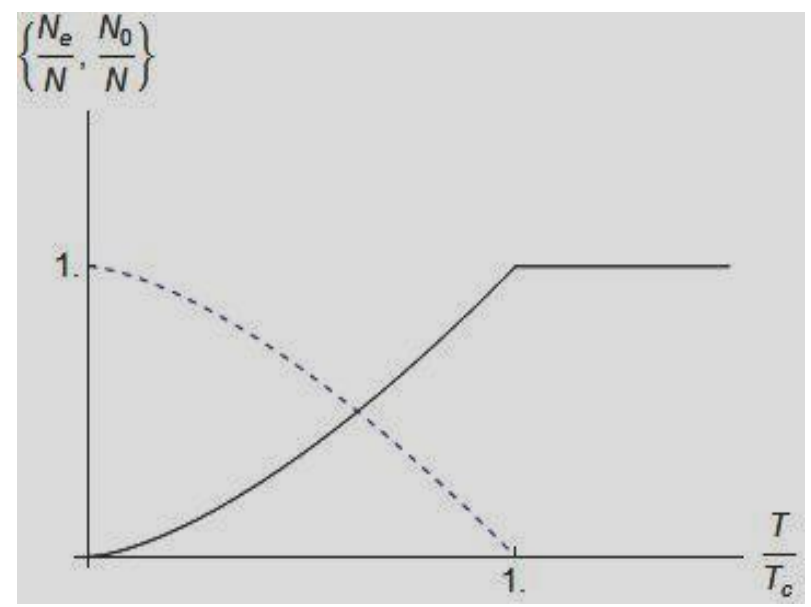

Fig. 3.1. Fracciones de la fase normal y fase condensada (línea punteada) en un gas ideal de Bose-Einstein como función del parámetro temperatura $\left(T / T_{c}\right)$ [6].

La Fig. 3.1 muestra la forma en la cual las fracciones $\left(N_{e} / N\right)$ y $\left(N_{0} / N\right)$ varía con la temperatura $T$. Para $T>T_{c}$, tenemos solo la fase normal; el número de partículas 
en el estado base en términos de la fugacidad $z /(1-z)$, el cual es completamente despreciable en comparación con el número total de partículas $N$. Notemos que, como $T \rightarrow T_{c}$ por abajo, la fracción condensada desaparece:

$$
\frac{N_{0}}{N}=1-\left(\frac{T}{T_{c}}\right)^{3 / 2} \approx \frac{3}{2} \frac{T_{c}-T}{T_{c}} .
$$

También nos interesa estudiar la variación de la fugacidad z con respecto a la temperatura $T$. Sin embargo, es más simple considerar la variación de z con respecto al parámetro $\left(v / \Lambda^{3}\right)$, el último será proporcional a $T^{3 / 2}$. Para $0 \leq\left(v / \Lambda^{3}\right) \leq(2.612)^{-1}$, el cual corresponde a $0 \leq T \leq T_{c}$, el parámetro $z \simeq 1$, ver la ecuación (3.26). Para $\left(v / \Lambda^{3}\right)>(2.612)^{-1}, z<1$ es determinado por la siguiente relación

$$
g_{3 / 2}(z)=\left(\Lambda^{3} / v\right)<2.612 .
$$

Para $\left(v / \Lambda^{3}\right) \gg 1$, tenemos: $g_{3 / 2}(z) \ll 1$ y, por lo tanto, $z \ll 1$. Bajo estas circunstancias, $g_{3 / 2}(z) \simeq z$, a primer orden en el desarrollo de la función de Bose-Einstein de $g_{3 / 2}(z)$, ver ecuación 3.12 . Por lo tanto, en está región, $z \simeq\left(v / \Lambda^{3}\right)^{-1}$, en concordancia con el caso clásico. La Fig. 3.2 muestra la variación de z con respecto al parámetro $\left(v / \Lambda^{3}\right)$. Ahora, examinaremos la variación de la presión $P$ con respecto a la temperatura $T$, manteniendo el volumen por partícula fijo $v$. Para $T<T_{c}$, la presión está dada por la ecuación (3.9), con z reemplazado por la unidad, es decir,

$$
P(T)=\frac{\kappa T}{\Lambda^{3}} \zeta\left(\frac{5}{2}\right)
$$

el cual es proporcional a $T^{5 / 2}$ y es independiente de $v$ implicando la compresibilidad infinita. En el punto de transición el valor de la presión es

$$
P\left(T_{c}\right)=\left(\frac{2 \pi m}{h^{2}}\right)^{3 / 2} \kappa T_{c}^{5 / 2} \zeta\left(\frac{5}{2}\right)
$$




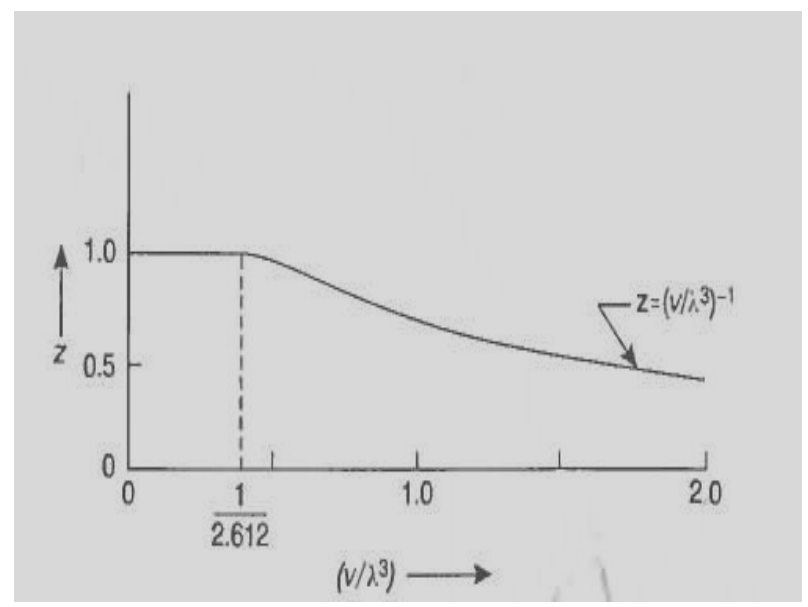

Fig. 3.2. La fugacidad de un gas ideal de Bose-Einstein como función de $\left(v / \Lambda^{3}\right)$ [46].

con ayuda de (3.27), está puede ser escrita como

$$
P\left(T_{c}\right)=\frac{\zeta\left(\frac{5}{2}\right)}{\zeta\left(\frac{3}{2}\right)}\left(\frac{N}{V} \kappa T_{c}\right) \simeq 0.5134\left(\frac{N}{V} \kappa T_{c}\right) .
$$

Aquí, la presión (ejercida por las partículas) de una gas ideal de Bose-Einstein en la temperatura de transición $T_{c}$ es, aproximadamente, la mitad de la presión (ejercida por las partículas) equivalente a un gas de Boltzmann. Para $T>T_{c}$, la presión está dada por

$$
P=\frac{N}{V} \kappa T \frac{g_{5 / 2}(z)}{g_{3 / 2}(z)}
$$

donde $z(T)$ es determinado por la siguiente relación

$$
g_{3 / 2}(z)=\frac{\Lambda^{3}}{v}=\frac{N}{V} \frac{\hbar^{3}}{(2 \pi m \kappa T)^{3 / 2}} .
$$

Al menos que la temperatura sea muy alta, la expresión para $P$ no puede ser escrita en una forma más simple, de hecho, para $T \gg T_{c}$, el desarrollo del virial (3.16) puede ser usado. Como $T \rightarrow \infty$, la presión se aproxima al valor clásico $N \kappa T / V$. Todas 
estas características se muestran en la Fig. 3.3. La línea de transición en la figura representa la ecuación (3.30). La curva exacta de $(P, T)$ sigue esta línea de $T=0$ hasta $T=T_{c} \mathrm{y}$, posteriormente, se aparta, tendiendo asintóticamente al límite clásico. Cabe señalar que la región a la derecha de la línea de transición pertenece solo a la fase normal, la línea de transición en si misma pertenece a la fase mixta, mientras que la región de la izquierda es inaccesible para el sistema. De la ecuación

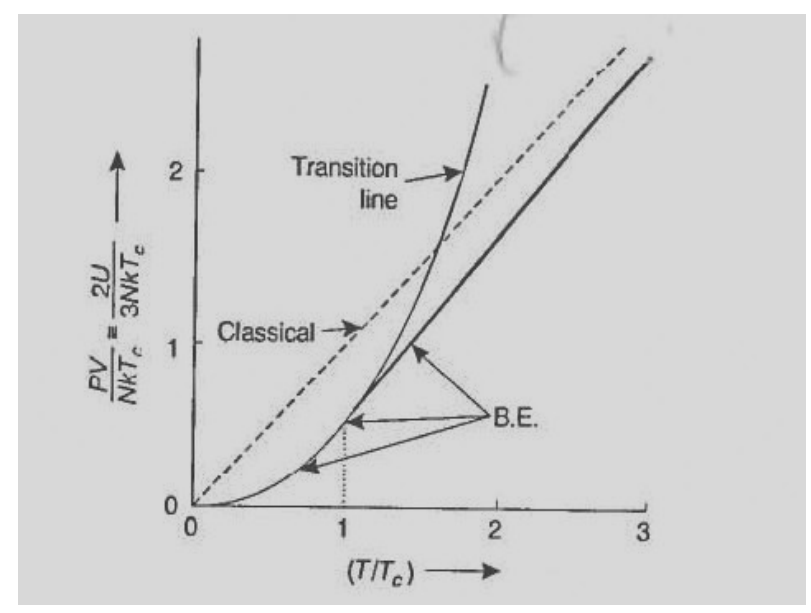

Fig. 3.3. La presión y la energía interna de un gas ideal de Bose-Einstein como función del parámetro de temperatura $\left(T / T_{c}\right)$ [6].

(3.14) se observa que hay una relación entre la energía interna del gas $U$ y la presión del gas $P$, por lo tanto, en la Fig. 3.3 también se describe la variación de la energía interna $U$ respecto a la temperatura $T$ (fijando $v$ ). Su pendiente, por consiguiente, debería ser una medida del calor específico $C_{V}(T)$ del gas. El calor específico tiende a cero a bajas temperaturas, y crece si $T$ aumenta hasta alcanzar su máximo valor en $T=T_{c}$; a partir de aquí comienza a disminuir, tendiendo asintóticamente a su valor clásico. Analíticamente para $T \leq T_{c}$, obtenemos: 


$$
\frac{C_{V}}{N \kappa}=\frac{3}{2} \frac{V}{N} \zeta\left(\frac{5}{2}\right) \frac{d}{d T}\left(\frac{T}{\Lambda^{3}}\right)=\frac{15}{4} \zeta\left(\frac{5}{2}\right) \frac{v}{\Lambda^{3}}
$$

el cual es proporcional a $T^{3 / 2}$. En $T=T_{c}$, tenemos:

$$
\frac{C_{V}\left(T_{c}\right)}{N \kappa}=\frac{15}{4} \frac{\zeta\left(\frac{5}{2}\right)}{\zeta\left(\frac{3}{2}\right)} \simeq 1.925
$$

el cual es considerablemente más alto que el valor clásico de $\frac{3}{2}$. Para $T>T_{c}$, obtenemos una formula absoluta. Ante todo,

$$
\frac{C_{V}}{N \kappa}=\left[\frac{\partial}{\partial T}\left(\frac{3}{2} T \frac{g_{5 / 2}(z)}{g_{3 / 2}(z)}\right)\right]_{v},
$$

Para realizar la derivada, necesitamos saber $(\partial z / \partial T)_{v}$, esto puede ser obtenido de las ecuaciones 3.13 y 3.29 con ayuda de la siguiente relación:

$$
z \frac{\partial}{\partial z}\left[g_{\nu}\right] \equiv \frac{\partial}{\partial(\ln z)} g_{\nu}(z)=g_{\nu-1}(z) .
$$

Por un lado, ya que $g_{3 / 2}(z) \propto T^{-3 / 2}$,

$$
\left[\frac{\partial}{\partial T} g_{3 / 2}(z)\right]_{v}=-\frac{3}{2 T} g_{3 / 2}(z)
$$

por el otro,

$$
z \frac{\partial}{\partial z} g_{3 / 2}(z)=g_{1 / 2}(z)
$$

Combinando los dos últimos resultados, obtenemos

$$
\frac{1}{z}\left(\frac{\partial z}{\partial T}\right)_{v}=-\frac{3}{2 T} \frac{g_{3 / 2}(z)}{g_{1 / 2}(z)}
$$

La ecuación (3.37) ahora da

$$
\frac{C_{V}}{N \kappa}=\frac{15}{4} \frac{g_{5 / 2}(z)}{g_{3 / 2}(z)}-\frac{g_{3 / 2}(z)}{g_{1 / 2}(z)},
$$


el valor de z como una función de $T$ está otra vez determinado por la ecuación (3.29). En el límite $z \rightarrow 1$, el segundo término en la ecuación (3.42) desaparece a causa de la divergencia de la función de Bose-Einstein $g_{1 / 2}(z)$, mientras el primer término da el resultado exacto que aparece en la ecuación (3.36). El calor específico es una función continua en el punto de transición, su derivada, por otro lado, no es continua en este punto. La magnitud de esta discontinuidad es

$$
\left(\frac{\partial C_{V}}{\partial T}\right)_{T=T_{c}-0}-\left(\frac{\partial C_{V}}{\partial T}\right)_{T=T_{c}+0}=\frac{27 N \kappa}{16 \pi T_{c}}\left\{\zeta\left(\frac{3}{2}\right)\right\}^{2} \simeq 3.665 \frac{N \kappa}{T_{c}} .
$$

Para $T>T_{c}$, el calor específico disminuye de manera constante hacia el valor límite

$$
\left(\frac{C_{V}}{N \kappa}\right)_{z \rightarrow 0}=\frac{15}{4}-\frac{9}{4}=\frac{3}{2}
$$

En la Fig. 3.4 se muestran todas estas características del calor específico como función de la temperatura $\left(C_{V}, T\right)$. Esta curva es muy similar a la obtenida con el experimento para helio líquido $\mathrm{He}^{4}$ ver Fig. 3.5. En 1938, F. London [65] sugiere, la transición de fase que ocurre en el helio líquido $H e^{4}$ a una temperatura alrededor $2.19 \mathrm{~K}$ podría ser una manifestación del condensado de Bose-Einstein que toma lugar en líquido. Si sustituimos en la ecuación (3.27), datos para el helio líquido $H e^{4}$, para $m=6.65 \times 10^{-24} \mathrm{~g}$ y $V=27.6 \mathrm{~cm}^{3} / \mathrm{mol}$, obtenemos para $T_{c}$ un valor aproximado de $3.13 \mathrm{~K}$, el cual no es tan diferente al valor observado en la temperatura de transición del líquido. 


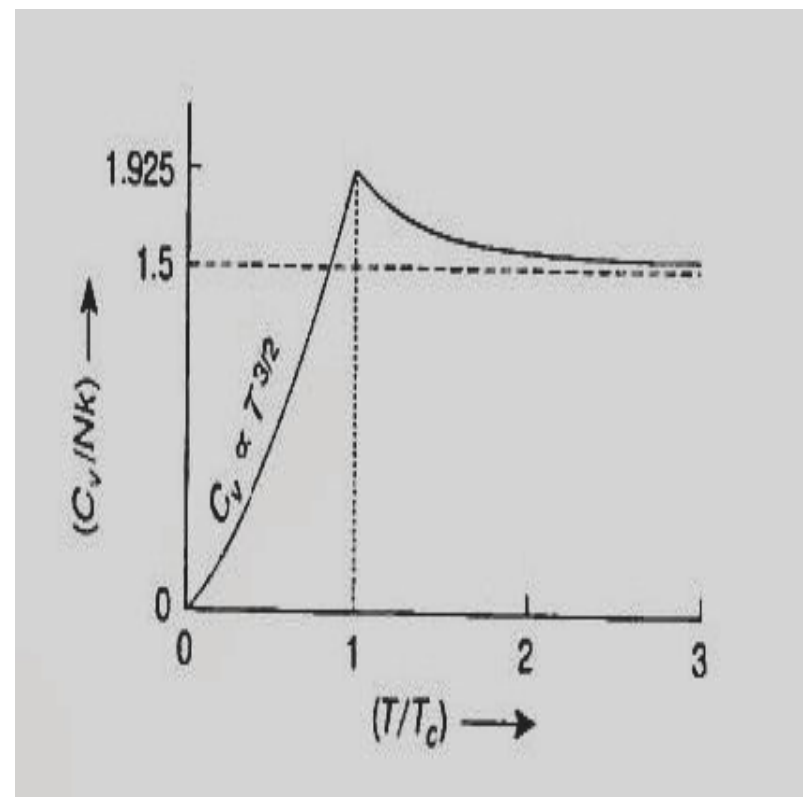

Fig. 3.4. El calor específico de un gas ideal de Bose-Einstein como función del parámetro de temperatura $\left(T / T_{c}\right)$ [4].

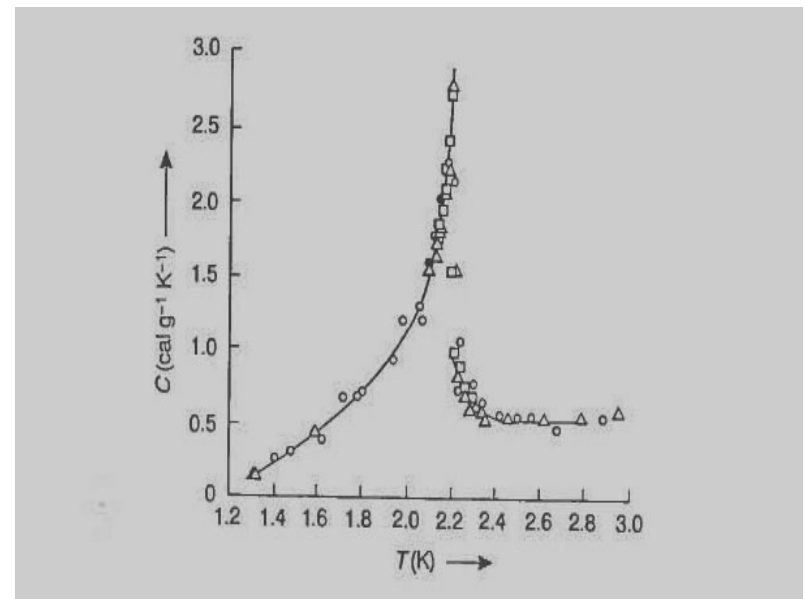

Fig. 3.5. El calor específico del helio líquido $H e^{4}$ bajo su propia presión de vapor [6]. 
CApítulo 4

\section{Condensación de Bose Einstein en un campo gravitacional homogéneo}

La idea central del trabajo es verificar el PEE desde un punto de vista meramente cuántico. Esto se hará por medio de una propuesta experimental, la cual consiste en realizar el condensado de Bose-Einstein con átomos del isótopo del Rubidio- $87{ }^{87} R b$ en un sistema en caída libre. Para ser más precisos, la propuesta experimental se realizará en la torre de la Universidad de Bremen en Alemania, la cual tiene una altura de $146 m$ (con una caída libre efectiva de $110 \mathrm{~m}$ ) con una calidad de ingravidez de $10^{-5} \frac{\mathrm{m}}{\mathrm{s}^{2}}$ durante 4.7 segundos [66]. En la torre de Bremen se dejará caer una cápsula con una altura total de $2.86 \mathrm{~m}$, dentro de esta cápsula se colocará todo el dispositivo experimental para lograr el condensado de Bose-Einstein junto con el dispositivo encargado de obtener los parámetros termodinámicos a considerar. Vale la pena mencionar que primero se logra el condensado y después se deja caer la cápsula con el condensado. Para verificar el PEE al realizar el condensado de Bose-Einstein en presencia de un campo gravitacional homogéneo en caída libre, estudiaremos que parámetro termodinámico es el más adecuado para medir en el laboratorio. Todo apunta que la temperatura de con- 
densación es buen parámetro termodinámico para comparar las mediciones hechas en un laboratorio con los resultados obtenidos en el experimento de la Universidad de Bremen, así poner a prueba el PEE.

Primero consideremos un gas de Bose-Einstein encerrado en un recipiente de volumen $V$, y se calcularan sus propiedades termodinámicas como la presión del gas, el numero de partículas promedio como función de la temperatura, y la temperatura de condensación. Este problema nos ayudara a entender como se ven modificadas las propiedades termodinámicas del gas cuando se toma en cuenta un campo gravitacional homogéneo. En la segunda parte se calcularan la propiedades termodinámicas del gas de Bose-Einstein cuando un campo gravitacional homogéneo este presente, pero a diferencia del problema anterior, el gas ya no estará encerrado en un recipiente de volumen finito, más bien, estará confinado por medio de osciladores armónicos anisotrópicos

\subsection{Gas ideal de Bose-Einstein confinado en un recipiente}

\subsubsection{Niveles de Energía}

Para obtener la temperatura de condensación consideraremos un gas encerrado en un volumen $V$ de altura $L$ con $N$ partículas indistinguibles que no interactúan entre si, en presencia de un campo gravitacional homogéneo que solo actúa en la dirección $z$ con condiciones de frontera tipo Dirichlet $\Psi(z=0)=0$ y $\Psi(z=L)=0$ (donde $\Psi$ es la función de onda) . La altura del contenedor también se encuentra en la dirección z, y a la hora de resolver el problema de valores propios solo la dirección $z$ se verá afectada por la presencia del campo gravitacional y lo que pase en el plano (formado por los ejes $x-y$ ) será independiente al eje $z$. Por lo tanto, 
en el plano se tendrá una partícula libre confinada en una caja y en la dirección z una partícula inmersa en un potencial gravitacional homogéneo.

El problema de una partícula inmersa en un campo gravitacional homogéneo, donde se considera que la función de onda se anula en $\pm \infty$, ya ha sido resuelto en muchos libros de mecánica cuántica [67]. Las funciones de onda son las funciones Airy. Debido a que nuestro problema requiere un recipiente finito, no podemos considerar estas soluciones de la ecuación de Schrödinger. Como se mencionó anteriormente, es independiente lo que pasa en el plano y lo que pasa en el eje z. En el plano se tiene el problema de una partícula encerrada en una caja en ausencia de campo gravitacional, el cual se resuelve de la manera usual haciendo uso de la ecuación de Schrödinger en dos dimensiones, mientras que en el eje $z$, se tiene una partícula en una barrera de potencial en presencia de un campo gravitacional homogéneo el cual se resolverá recurriendo a una teoría de perturbaciones independiente del tiempo (ver apéndice B). Consideraremos el Hamiltoniano sin perturbar de una partícula libre y el campo gravitacional homogéneo como la perturbación.

Recordemos, los valores propios de energía se obtienen al resolver la ecuación de Schrödinger para una partícula libre de masa m dentro de una barrera de potencial.

$$
V(\mathbf{z})= \begin{cases}0, & \text { para } \mathbf{0}<\mathbf{z}<\mathbf{L} \\ \infty, & \text { en cualquier otro lado }\end{cases}
$$

Los valores propios de energía correspondientes al Hamiltoniano sin perturbar son 68]:

$$
\varepsilon_{n}^{0}=\frac{\hbar^{2} \pi^{2}}{2 m L^{2}} n^{2} . \quad(\sin \text { perturbar })
$$

También, la función de onda asociada es: 


$$
\psi(z)=\sqrt{\frac{2}{L}} \sin \left(\frac{n \pi z}{L}\right) .
$$

Utilizando el siguiente desarrollo a segundo orden, obtendremos los valores propios de energía para una partícula inmersa en un campo gravitacional homogéneo.

$$
\triangle_{n}=\lambda V_{n n}+\lambda^{2} \sum_{n^{\prime} \neq n=1}^{\infty} \frac{\left|V_{n n^{\prime}}\right|^{2}}{\varepsilon_{n^{\prime}}^{(0)}-\varepsilon_{n}^{(0)}}+\ldots
$$

Este último desarrollo tiene sentido si solo si la serie converge. Primero calcularemos los elementos de matriz de $V_{n n^{\prime}}$ donde $n \neq n^{\prime}$

$$
V_{n n^{\prime}}=\left\langle n^{(0)}|V(z)| n^{(0)^{\prime}}\right\rangle=\int_{0}^{L} \psi^{*}(z) V(z) \psi(z) d z .
$$

Sustituyendo $V(z)=m g z$ y $\psi(z)$ obtenemos:

$$
V_{n n^{\prime}}=\frac{2 m g}{L} \int_{0}^{L} z \sin \left(\frac{n \pi z}{L}\right) \sin \left(\frac{n^{\prime} \pi z}{L}\right) d z ; \quad n^{\prime} \neq n .
$$

Utilizamos las siguientes propiedades trigonométricas para reescribir la ultima ecuación,

$$
\begin{aligned}
& \cos (A+B)=\cos (A) \cos (B)-\sin (A) \sin (B) \\
& \cos (A-B)=\cos (A) \cos (B)+\sin (A) \sin (B)
\end{aligned}
$$

Substituyendo 4.6 en 4.5 vemos que el integrando se expresa como,

$$
\sin \left(\frac{n \pi z}{L}\right) \sin \left(\frac{n^{\prime} \pi z}{L}\right)=\frac{1}{2} \cos \left[\left(n-n^{\prime}\right) \frac{\pi z}{L}\right]-\cos \left[\left(n+n^{\prime}\right) \frac{\pi z}{L}\right]
$$

para $n^{\prime} \neq n$ y $n^{\prime}, n=0,1,2,3, \ldots$ Entonces,

$$
V_{n n^{\prime}}=\frac{2 m g}{2 L}\left\{\int_{0}^{L} z \cos \left[\left(n-n^{\prime}\right) \frac{\pi z}{L}\right] d z-\int_{0}^{L} z \cos \left[\left(n+n^{\prime}\right) \frac{\pi z}{L}\right] d z\right\}
$$


integrando por partes se obtiene que

$$
V_{n n^{\prime}}=\frac{m g}{L}\left\{-\frac{L}{\pi\left(n-n^{\prime}\right)} \int_{0}^{L} \sin \left[\left(n-n^{\prime}\right) \frac{\pi z}{L}\right] d z+\frac{L}{\pi\left(n+n^{\prime}\right)} \int_{0}^{L} \sin \left[\left(n+n^{\prime}\right) \frac{\pi z}{L}\right] d z\right\},
$$

y, consecuentemente,

$$
V_{n n^{\prime}}=\frac{m L}{\pi^{2}}\left\{\frac{\cos \left[\left(n-n^{\prime}\right) \pi\right]-1}{\left(n-n^{\prime}\right)^{2}}-\frac{\cos \left[\left(n+n^{\prime}\right) \pi\right]-1}{\left(n+n^{\prime}\right)^{2}}\right\} ; \quad n \neq n^{\prime} .
$$

Aquí tenemos dos posibilidades que $n-n^{\prime}$ sea par al igual que $n+n^{\prime}$ o que $n-n^{\prime}$ y $n+n^{\prime}$ sean impares.

1. Primera posibilidad que $n-n^{\prime} \mathbf{y} n+n^{\prime}$ sean pares.

$$
\begin{gathered}
n-n^{\prime}=2 q \quad n, n^{\prime}, q=1,2,3 \ldots \\
n^{\prime}=n-2 q \\
n+n^{\prime}=n+n-2 q \\
n+n^{\prime}=2(n-q) \quad \text { es par } \\
V_{n n^{\prime}}=\frac{m g L}{\pi^{2}}\left\{\frac{\cos [2 q \pi]-1}{(2 q)^{2}}-\frac{\cos [2(n-q) \pi]-1}{[2(n-q)]^{2}}\right\}, \\
\quad \cos [2 q \pi]=1=\cos [2 n \pi], \\
\sin [2 q \pi]=0=\sin [2 n \pi] . \\
V_{n n^{\prime}}=\frac{m g L}{\pi^{2}}\left\{\frac{1-1}{(2 q)^{2}}-\frac{1-1}{[2(n-q)]^{2}}\right\}=0 .
\end{gathered}
$$

2. Segunda posibilidad que $n-n^{\prime} \mathbf{y} n+n^{\prime}$ sean impares. 


$$
\begin{aligned}
& n-n^{\prime}=2 q+1 \quad n \neq n^{\prime}=1,2,3 \ldots \\
& n^{\prime}=n-2 q-1 \quad q=0,1,2,3 \ldots \\
& n+n^{\prime}=n+n-2 q-1 \\
& n+n^{\prime}=2(n-q)-1 \quad \text { es impar } \\
& V_{n n^{\prime}}=\frac{m g L}{\pi^{2}}\left\{\frac{\cos [(2 q+1) \pi]-1}{(2 q+1)^{2}}-\frac{\cos [(2(n-q)-1) \pi]-1}{[2(n-q)-1]^{2}}\right\}, \\
& V_{n n^{\prime}}=\frac{-8 m g L}{\pi^{2}}\left\{\frac{n^{2}-n(2 q+1)}{(2 q+1)^{2}[2 n-(2 q+1)]^{2}}\right\} .
\end{aligned}
$$

Ahora calculemos $\left|V_{n n^{\prime}}\right|^{2}$

$$
\left|V_{n n^{\prime}}\right|^{2}=\frac{64 m^{2} g^{2} L^{2}}{\pi^{4}}\left(\frac{n^{4}-n^{2}(2 q-1)[2 n-(2 q-1)]}{(2 q+1)^{4}[2 n-(2 q+1)]^{4}}\right) .
$$

Los valores propios de energía de "partícula libre"son:

$$
\varepsilon_{n}^{0}=\frac{\hbar^{2} \pi^{2}}{2 m L^{2}} n^{2}
$$

Para la diferencia de energías $\varepsilon_{n}^{0}-\varepsilon_{n^{\prime}}^{0}$ obtenemos:

$$
\begin{gathered}
\varepsilon_{n}^{0}-\varepsilon_{n^{\prime}}^{0}=\frac{\hbar^{2} \pi^{2}}{2 m L^{2}}\left[2 n(2 q+1)-(2 q+1)^{2}\right] ; \\
\sum_{n^{\prime} \neq n=1}^{\infty} \frac{\left|V_{n n^{\prime}}\right|^{2}}{\varepsilon_{n}^{(0)}-\varepsilon_{n^{\prime}}^{(0)}} .
\end{gathered}
$$

Sustituyendo el valor de $\left|V_{n n^{\prime}}\right|^{2}$ y la diferencia de energías $\varepsilon_{n}^{0}-\varepsilon_{n^{\prime}}^{0}$ en la ecuación anterior se obtiene:

$$
\sum_{n^{\prime} \neq n=1}^{\infty} \frac{\left|V_{n n^{\prime}}\right|^{2}}{\varepsilon_{n}^{(0)}-\varepsilon_{n^{\prime}}^{(0)}}=\sum_{q=0}^{\infty} \frac{128 m^{3} g^{2} L^{4}}{\hbar^{2} \pi^{6}}\left[\frac{n^{4}-n^{2}(2 q+1)[2 n-(2 q+1)]}{(2 q+1)^{5}[2 n-(2 q+1)]^{5}}\right],
$$




$$
\begin{aligned}
\sum_{n^{\prime} \neq n=1}^{\infty} \frac{\left|V_{n n^{\prime}}\right|^{2}}{\varepsilon_{n}^{(0)}-\varepsilon_{n^{\prime}}^{(0)}} & =\frac{128 m^{3} g^{2} L^{4}}{\hbar^{2} \pi^{6}} n^{4} \sum_{q=0}^{\infty} \frac{1}{(2 q+1)^{5}[2 n-(2 q+1)]^{5}} \\
& -\frac{128 m^{3} g^{2} L^{4}}{\hbar^{2} \pi^{6}} n^{2} \sum_{q=0}^{\infty} \frac{1}{(2 q+1)^{4}[2 n-(2 q+1)]^{4}}
\end{aligned}
$$

Todo se reduce a demostrar que estas series convergen

$$
\begin{aligned}
& \sum_{q=0}^{\infty} \frac{1}{(2 q+1)^{5}[2 n-(2 q+1)]^{5}}, \\
& \sum_{q=0}^{\infty} \frac{1}{(2 q+1)^{4}[2 n-(2 q+1)]^{4}} .
\end{aligned}
$$

La demostración de la convergencia de estas series se encuentra en el Apéndice C. Por lo tanto el método de teoría de perturbaciones está bien fundamentado. Ahora encontraremos un valor aproximado para las series.

Regresando la ecuación 4.24

$$
\sum_{q=0}^{\infty} \frac{1}{(2 q+1)^{5}[2 n-(2 q+1)]^{5}} \approx \frac{1}{32 n^{5}} \sum_{q=0}^{n-1} \frac{1}{(2 q+1)^{5}} .
$$

Aquí se utiliza una segunda aproximación

$$
\sum_{q=0}^{n-1}\left|\frac{1}{(2 q+1)^{5}}\right| \geq \sum_{q=n}^{\infty} \mid \frac{1}{(2 q+1)^{5}[2 n-(2 q+1)]^{5}} .
$$

Para la otra serie se tiene la siguiente aproximación

$$
\sum_{q=0}^{n} \frac{1}{(2 q+1)^{4}[2 n-(2 q+1)]^{4}} \approx \sum_{q=0}^{\infty} \frac{1}{16 n^{4}} \sum_{q=0}^{\infty} \frac{1}{(2 q+1)^{4}}
$$

Estas son las cotas superiores de nuestras series

$$
\sum_{q=0}^{n-1} \frac{1}{(2 q+1)^{5}} \leq \sum_{q=1}^{\infty} \frac{1}{q^{5}}=\zeta(5)
$$




$$
\sum_{q=0}^{n-1} \frac{1}{(2 q+1)^{4}} \leq \sum_{q=0}^{\infty} \frac{1}{2 q+1^{4}}=\frac{\pi^{4}}{96} .
$$

Finalmente, el resultado de las dos series de manera aproximada es:

$$
\begin{gathered}
\sum_{q=0}^{\infty} \frac{1}{(2 q+1)^{5}[2 n-(2 q+1)]^{5}} \approx \frac{1}{32 n^{5}} \frac{31}{32} \zeta(5), \\
\sum_{q=0}^{\infty} \frac{1}{(2 q+1)^{4}[2 n-(2 q+1)]^{4}} \approx \frac{1}{16 n^{4}} \frac{\pi^{4}}{96} .
\end{gathered}
$$

Recordando el segundo término de la ecuación del desarrollo en teoría de perturbaciones para el desplazamiento de la energía:

$$
\begin{aligned}
\sum_{n^{\prime} \neq n}^{\infty} \frac{\left|V_{n n^{\prime}}\right|^{2}}{E_{n}^{0}-E_{n^{\prime}}^{0}} & \geq \frac{128 m^{3} g^{2} L^{4}}{\hbar^{2} \pi^{6}} n^{4} \sum_{q=0}^{\infty} \frac{1}{(2 q+1)^{5}[2 n-(2 q+1)]^{5}} \\
& -\frac{128 m^{3} g^{2} L^{4}}{\hbar^{2} \pi^{6}} \sum_{q=0}^{\infty} n^{2} \frac{1}{(2 q+1)^{4}[2 n-(2 q+1)]^{4}} .
\end{aligned}
$$

Sustituyendo el valor de las series se obtiene:

$$
\sum_{n^{\prime} \neq n}^{\infty} \frac{\left|V_{n n^{\prime}}\right|^{2}}{E_{n}^{0}-E_{n^{\prime}}^{0}} \geq \frac{4 m^{3} g^{2} L^{4}}{\hbar^{2} \pi^{6}}\left(\frac{31}{32} \frac{\zeta(5)}{n}-\frac{\pi^{4}}{48} \frac{1}{n^{2}}\right) .
$$

En la ecuación 4.34, $\zeta(5)=1.037$ y $\frac{\pi^{4}}{48}=2.02936$ Ahora calcularemos el primer término del desarrollo del desplazamiento de la energía en teoría de perturbaciones a segundo orden. Los elementos de matriz $V_{n n}=\langle n|V(z)| n\rangle$ se obtienen de la siguiente forma:

$$
V_{n n}=\int_{0}^{L} m g z \sin \left(\frac{n \pi z}{L}\right) \sin \left(\frac{n \pi z}{L}\right) d z=\int_{0}^{L} m g z \sin \left(\frac{n \pi z}{L}\right)^{2} d z .
$$

Usando la siguiente identidad trigonométrica $\sin \left(\frac{n \pi z}{L}\right)^{2}=\frac{1}{2}-\frac{1}{2} \cos \left(\frac{2 n \pi z}{L}\right)$ se obtiene: 


$$
V_{n n}=\int_{0}^{L} m g z\left(\frac{1}{2}-\frac{1}{2} \cos \left(\frac{2 n \pi z}{L}\right)\right) d z .
$$

Al integrar por partes la segunda integral se obtiene como resultado una función par que al evaluarla da cero, por lo tanto, el resultado proviene del primer término de la ecuación anterior:

$$
V_{n n}=\frac{m g L}{2}
$$

Sustituyendo los elementos de matriz de primer y segundo orden obtenidos en el desarrollo del desplazamiento de la energía, dado por,

$$
\triangle_{n}=\varepsilon_{n}-\varepsilon_{n}^{(0)}=\lambda V_{n n}+\lambda^{2} \sum_{n^{\prime} \neq n}^{\infty} \frac{\left|V_{n n^{\prime}}\right|^{2}}{\varepsilon_{n}^{(0)}-\varepsilon_{n^{\prime}}^{(0)}}+\ldots
$$

se obtiene que,

$$
\varepsilon_{n}=\frac{\hbar^{2} \pi^{2}}{2 m L^{2}} n^{2}+\lambda \frac{m g L}{2}+\lambda^{2} \frac{4 m^{3} g^{2} L^{4}}{\hbar^{2} \pi^{6}}\left(\frac{\zeta(5)}{n}-\frac{\pi^{4}}{48} \frac{1}{n^{2}}\right) .
$$

Para $\lambda=1$ (la perturbación máxima) la ecuación 4.34 se reduce a la de los valores propios de energía para un gas de Bose-Einstein encerrado en un recipiente de volumen $V$ en presencia de una campo gravitacional homogéneo.

$$
\varepsilon_{n}=\frac{\hbar^{2} \pi^{2}}{2 m L^{2}} n^{2}+\frac{m g L}{2}+\frac{4 m^{3} g^{2} L^{4}}{\hbar^{2} \pi^{6}}\left(\frac{\zeta(5)}{n}-\frac{\pi^{4}}{48} \frac{1}{n^{2}}\right) .
$$

\subsubsection{Temperatura de condensación en un campo gravitacional homogéneo}

Con los valores propios de energía obtenidos en la sección anterior al recurrir a la teoría de perturbaciones independiente del tiempo para un gas de Bose-Einstein encerrado en un recipiente de volumen finito con partículas que no interactúan entre si en presencia de un campo gravitacional homogéneo, calcularemos el número de partículas promedio en el recipiente y la temperatura de condensación. 
Recurriendo a la función de partición del conjunto gran canónico para un gas de Bose-Einstein podemos escribir el número de partículas promedio de la siguiente forma:

$$
N=\sum_{\varepsilon}\left\langle n_{\varepsilon}\right\rangle=\sum_{\varepsilon} \frac{1}{z^{-1} e^{\beta \varepsilon}-1},
$$

donde $\beta=\frac{1}{\kappa T}$ y $z$ es la fugacidad del gas $z=e^{\frac{\mu}{\kappa T}}$.

Como hemos considerado de manera independiente el comportamiento en el plano $(x-y)$ respecto a lo que sucede en la dirección $z$, debido a que el campo gravitacional solo afecta al gas en la dirección z, podemos separar la energía en dos contribuciones, la del plano $\varepsilon_{A}$ (donde $\left.A(x, y)\right)$ y la correspondiente a la dirección $\mathrm{Z} \varepsilon_{z}$.

La contribución de la energía en el plano $\varepsilon_{A}$ es independiente a la contribución de la energía en la dirección z, por lo cual podemos separar la sumatoria de la ecuación 4.41) en dos, como se muestra a continuación

$$
N=\frac{z}{1-z}+\sum_{\varepsilon_{A}=1}^{\infty} \sum_{\varepsilon_{z}=1}^{\infty} \frac{1}{z^{-1} e^{\beta\left[\varepsilon_{A}+\varepsilon_{z}\right]}-1} .
$$

Las partículas en el estado base están dadas por,

$$
N_{0}=\frac{z}{1-z} \text {. }
$$

La energía en el plano se obtiene de resolver la ecuación de Schrödinger de una partícula encerrada en una caja de longitud $b, \varepsilon_{A}=\frac{\hbar^{2} \pi^{2}}{2 m a^{2}}\left(n_{x}{ }^{2}+n_{y}{ }^{2}\right)$ y la densidad de estados por unidad de energía correspondiente a esta energía es $\Omega\left(\varepsilon_{A}\right)=\frac{m b^{2}}{2 \pi \hbar^{2}}$. Hagamos la aproximación al continuo de la suma de energías en el plano $\sum_{\varepsilon_{A}} \rightarrow$ $\int_{0}^{\infty} \Omega\left(\varepsilon_{A}\right) d \varepsilon_{A}$ y calculamos la integral correspondiente:

$$
N-N_{0}=\sum_{\varepsilon_{z}=1}^{\infty} \int_{0}^{\infty}\left(\frac{m b^{2}}{2 \pi \hbar^{2}}\right) \frac{1}{z^{-1} e^{\beta \varepsilon_{z}} e^{\beta \varepsilon_{A}}-1} d \varepsilon_{A},
$$




$$
N-N_{0}=-\frac{1}{\beta}\left(\frac{m b^{2}}{2 \pi \hbar^{2}}\right) \sum_{\varepsilon_{z}=1}^{\infty} \ln \left[1-z e^{-\beta \varepsilon_{z}}\right] .
$$

Ahora calculamos el número de partículas en los estados excitados, para lo cual recurrimos a los valores propios de energía encontrados en la sección anterior,

$$
\varepsilon_{z}=\frac{\hbar^{2} \pi^{2}}{2 m L^{2}} n^{2}+\frac{m g L}{2}
$$

Una vez más hacemos el cambio al continuo de la suma de las energías en la dirección z por la integral; cabe mencionar que la densidad de estados por unidad de energía es la unidad, debido que tenemos una partícula en una dimensión con estados ligados [69]. Entonces,

$$
N-N_{0}=-\frac{1}{\beta}\left(\frac{m b^{2}}{2 \pi \hbar^{2}}\right) \int_{0}^{\infty} \ln \left[1-z e^{-\beta\left[\frac{\hbar^{2} \pi^{2}}{2 m L^{2}} n^{2}+\frac{m g L}{2}\right]}\right] d n .
$$

Definimos $\gamma=z e^{-\frac{\beta m g L}{2}}$, con lo cual la ecuación 4.47 se transforma en,

$$
N-N_{0}=-\frac{1}{\beta}\left(\frac{m b^{2}}{2 \pi \hbar^{2}}\right) \int_{0}^{\infty} \ln \left[1-\gamma e^{-\beta\left(\frac{\hbar^{2} \pi^{2}}{2 m L^{2}}\right) n^{2}}\right] d n .
$$

Integrando por partes:

$$
N-N_{0}=V \frac{(2 \pi m \kappa T)^{\frac{3}{2}}}{h^{3}} g_{3 / 2}(\gamma)
$$

Aquí $g_{\nu}(z)$ son las funciones de Bose-Einstein [46]. Recordando que la longitud térmica de Broglie es $\Lambda=\frac{(2 \pi m \kappa T)^{\frac{1}{2}}}{h}$ y la definición de $\gamma=z e^{-\frac{m g L}{2 \kappa T}}$ se obtiene el número de partículas en los estados excitados como función del campo gravitacional

$$
N-N_{0}=\frac{V}{\Lambda^{3}} g_{3 / 2}\left(z e^{-\frac{m g L}{2 \kappa T}}\right)
$$


En el que no hay campo gravitacional, al tomar el límite $g \rightarrow 0$, recuperamos el valor clásico,

$$
\lim _{g \rightarrow 0} \frac{V}{\Lambda^{3}} g_{3 / 2}\left(z e^{-\frac{m g L}{2 \kappa T}}\right)=\frac{V}{\Lambda^{3}} g_{3 / 2}(z) .
$$

Este es el resultado del número de partículas en los estados excitados en ausencia de campo gravitacional. Ahora procederemos a calcular la temperatura de condensación en presencia de un campo gravitacional homogéneo, recordando que la energía potencial es menor a cualquier energía que este involucrada en el problema en particular la energía interna $\frac{m g L}{\kappa T}<1$. Para ello vamos a observar el comportamiento de la función de Bose-Einstein

$$
g_{3 / 2}\left(z e^{-\frac{m g L}{2 \kappa T}}\right)=g_{3 / 2}\left(e^{\frac{2 \mu-m g L}{2 \kappa T}}\right)
$$

Ahora, definimos un potencial químico efectivo $\mu_{e f f}=\mu-\frac{m g L}{2}$ el cual este acotado entre $\left(-\infty,-\frac{m g L}{2}\right)$.

Así pues,

$$
g_{3 / 2}\left(z e^{\frac{\mu_{e f f}}{\kappa T}}\right)=g_{3 / 2}\left(z e^{Z_{e f f}}\right)
$$

donde $Z_{e f f}=e^{\frac{2 \mu-m g L}{2 \kappa T}}$ es la fugacidad efectiva. El potencial químico efectivo tiene un valor límite que ya no es cero y este valor es $\frac{m g L}{2}$; si el campo gravitacional es nulo se recupera el valor límite igual a cero. Esto muestra que el potencial químico se vería modificado en presencia de campos externos, o al considerar interacción entre las partículas, por lo tanto, también la temperatura de condensación $T_{c}$ se verá modificada. Como lo que nos interesa es encontrar la temperatura de condensación y la función de Bose-Einstein está en función del campo gravitacional hay que invertir de alguna manera la función de Bose $g_{3 / 2}\left(z e^{Z_{e f f}}\right)$. Para ello se utilizará un desarrollo en serie de $g_{\nu} \approx\left(e^{-\alpha}\right)$. 


$$
g_{\nu} \approx\left(e^{-\alpha}\right)=\frac{\Gamma(1-\nu)}{\alpha^{1-\nu}}+\sum_{i=0}^{\infty} \frac{(-1)^{i}}{i !} \zeta(\nu-i) \alpha^{i} .
$$

Donde $\zeta(s)$ es la función zeta de Riemann analíticamente continua $\forall s \neq 1$ [46]. Para nuestro problema $\nu=3 / 2$ :

$$
\begin{gathered}
g_{3 / 2} \approx\left(e^{-\alpha}\right)=\frac{\Gamma(1-3 / 2)}{\alpha^{1-3 / 2}}+\sum_{i=0}^{\infty} \frac{(-1)^{i}}{i !} \zeta(3 / 2-i) \alpha^{i}, \\
g_{3 / 2} \approx\left(e^{-\alpha}\right) \approx \frac{\Gamma(-1 / 2)}{\alpha^{-1 / 2}}+\zeta(3 / 2),
\end{gathered}
$$

donde $-\ln \left(Z_{\text {eff }}\right)=\alpha$. Entonces,

$$
N_{e}=\frac{V}{\Lambda^{3}} g^{3 / 2}\left(Z_{e f f}\right) \approx \frac{V}{\Lambda^{3}}\left[\zeta\left(\frac{3}{2}\right)+\Gamma\left(-\frac{1}{2}\right) \alpha^{\frac{1}{2}}\right] .
$$

Por otro lado recordemos que el número de partículas en el estado excitado cuando no hay campo gravitacional es:

$$
\begin{gathered}
\frac{N_{e}}{V}=\frac{\zeta(3 / 2)}{\left(\Lambda_{c}^{o}\right)^{3}} \\
\frac{N_{e}}{V}=\frac{(2 \pi m \kappa)^{3 / 2} \zeta(3 / 2)\left(T_{c}^{0}\right)^{3 / 2}}{h^{3}} .
\end{gathered}
$$

donde $T_{c}^{0}$ es la temperatura de condensación sin campo gravitacional.

Ahora igualamos la ecuación (4.57), poniendo el valor explícito de $\alpha$, con la ecuación 4.59 de manera que,

$$
\begin{gathered}
\frac{(2 \pi m \kappa)^{3 / 2} \zeta(3 / 2)\left(T_{c}^{0}\right)^{3 / 2}}{h^{3}}=\frac{(2 \pi m \kappa)^{3 / 2} T_{c}^{3 / 2}}{h^{3}}\left[\zeta(3 / 2)-\sqrt{\frac{\pi m g L}{2 \kappa T_{c}}}\right], \\
\left(T_{c}^{o}\right)^{3 / 2}=T_{c}^{3 / 2}\left[1-\frac{1}{\zeta(3 / 2)} \sqrt{\frac{\pi m g L}{2 \kappa T_{c}}}\right] .
\end{gathered}
$$


De la última ecuación se puede observar que la temperatura de condensación para un gas de Bose-Einstein en un campo gravitacional es mayor que cuando no existe campo gravitacional. Vamos ahora a escribir la ecuación (4.61), la temperatura de condensación como una función de la gravedad, en términos de la altura del recipiente, la masa de las partículas, la constante de Boltzmann y la temperatura de la condensación cuando no hay campo gravitacional.

De la ecuación 4.61, invirtiendo la expresión y haciendo uso del desarrollo binomial, obtenemos que

$$
T_{c} \approx T_{c}^{o}\left[1+\frac{2}{3} \frac{1}{\zeta(3 / 2)} \sqrt{\frac{\pi m g L}{2 \kappa T_{c}^{o}}}\right],
$$

donde la temperatura de condensación en ausencia de campo gravitacional por la ecuación 4.59) se reescribe ahora como,

$$
T_{c}^{o}=\frac{h^{2}}{2 \pi m \kappa}\left(\frac{N}{V \zeta(3 / 2)}\right)^{2 / 3}
$$

\subsubsection{Presión de un gas de Bose-Einstein en un campo gravitacional homogéneo}

Obtengamos la presión para un gas de bosones en un campo gravitacional homogéneo. En el conjunto gran canónico la presión del gas esta dada por la siguiente expresión:

$$
\frac{P V}{\kappa T}=\ln \Xi=-\sum_{\varepsilon=0}^{\infty} \ln \left[1-z e^{-\beta \varepsilon}\right] .
$$

Antes de calcular la presión recordemos que en mecánica estadística debemos dar un peso estadístico unitario a cada estado no degenerado de partícula individual por lo cual hay que tomar el estado con energía cero fuera de la suma. 


$$
\frac{P V}{\kappa T}=-\sum_{\varepsilon=1}^{\infty} \ln \left[1-z e^{-\beta \varepsilon}\right]-\ln [1-z]
$$

Como y dijimos, hemos dividido la energía de partícula individual en dos partes, la energía del plano " $\varepsilon_{A}$ " y la energía en la dirección z " $\varepsilon_{z}$ ", por lo tanto, la suma también la dividiremos en dos partes $\sum_{\varepsilon_{A}=1}^{\infty} \sum_{\varepsilon_{z}=1}^{\infty}$ por lo cual, la ecuación 4.65 se puede expresar de la siguiente forma:

$$
\frac{P V}{\kappa T}=-\sum_{\varepsilon_{A}=1}^{\infty} \sum_{\varepsilon_{z}=1}^{\infty} \ln \left[1-z e^{-\beta\left[\varepsilon_{A}+\varepsilon_{z}\right]}\right]-\ln [1-z] .
$$

Haciendo la aproximación al continuo considerando que la diferencia entre dos estados sucesivos de energía es pequeña,

$$
\sum_{\varepsilon_{A}=1}^{\infty} \rightarrow \int_{0}^{\infty} \Omega\left(\varepsilon_{A}\right) d \varepsilon_{A}
$$

donde la densidad de estados por unidad energía es $\Omega\left(\varepsilon_{A}\right)=\frac{m b^{2}}{2 \pi \hbar^{2}}$. Entonces:

$$
\sum_{\varepsilon_{A}=1}^{\infty} \rightarrow \int_{0}^{\infty}\left(\frac{m b^{2}}{2 \pi \hbar^{2}}\right) d \varepsilon_{A}
$$

Por lo tanto:

$$
\frac{P V}{\kappa T}=-\sum_{\varepsilon_{z}=1}^{\infty}\left(\frac{m b^{2}}{2 \pi \hbar^{2}}\right) \int_{0}^{\infty} \ln \left[1-w e^{-\beta \varepsilon_{A}}\right] d \varepsilon_{A}-\ln [1-z],
$$

donde $w=z e^{-\beta \varepsilon_{z}}$. Ahora calculemos la siguiente integral:

$$
I=\int_{0}^{\infty} \ln \left[1-w e^{-\beta \varepsilon_{A}}\right] d \varepsilon_{A} .
$$

Integrando por partes y sustituyendo el valor de $w$, obtenemos que,

$$
\frac{P V}{\kappa T}=\frac{1}{\beta}\left(\frac{m b^{2}}{2 \pi \hbar^{2}}\right) \sum_{r=1}^{\infty} \frac{z^{r}}{r^{2}} \sum_{\varepsilon_{z}=1}^{\infty} e^{-r \beta \varepsilon_{z}}-\ln [1-z] .
$$


Vamos a utilizar los valores propios de energía encontrados al usar teoría de perturbaciones independiente del tiempo a primer orden:

$$
\varepsilon_{z}=\frac{\hbar^{2} \pi^{2}}{2 m L} n^{2}+\frac{m g L}{2}
$$

Haremos el cambio al continuo de la suma a la integral.

$$
\sum_{n=1}^{\infty} \rightarrow \int_{0}^{\infty} \Omega\left(\varepsilon_{z}\right) d n
$$

donde por el hecho de que se tiene un problema de estados ligados en una dimensión la densidad de estados por unidad de energía es igual a uno, $\Omega\left(\varepsilon_{z}\right)=1$.

$$
\sum_{\varepsilon_{z}=1}^{\infty} e^{-r \beta \varepsilon_{z}}=\int_{0}^{\infty} e^{-\frac{r \beta m g L}{2}} e^{-\frac{r \beta \hbar^{2} \pi^{2}}{2 m L^{2}}} d n
$$

Resolviendo la integral y recordando que el volumen del contenedor es $V=b^{2} L$ se obtiene finalmente que

$$
\frac{P V}{\kappa T}=\frac{V}{\Lambda^{3}} g_{5 / 2}\left(z e^{-\frac{m g L}{2 \kappa T}}\right)
$$

la presión de un gas de bosones en un campo gravitacional homogéneo. Si hacemos tender $g \rightarrow 0$ recobramos el caso usual en la cual no hay campo gravitacional.

$$
\frac{P V}{\kappa T}=\frac{V}{\Lambda^{3}} g_{5 / 2}(z)
$$

\subsection{Gas ideal de Bose-Einstein confinado por osciladores armónicos anisotrópicos}

Consideremos un sistema de $N$ partículas inmersas en un potencial de oscilador armónico anisotrópico en tres dimensiones y un campo gravitacional homogéneo. Entonces, la energía potencial esta dada por, 


$$
U(x, y, z)=\frac{1}{2}\left(k_{1} x^{2}+k_{2} y^{2}+k_{3} z^{2}\right)+m g z
$$

Se define la frecuencia del oscilador armónico de la siguiente forma

$$
w_{i}=\frac{k_{i}}{m} ; \quad i=x, y, z
$$

de manera que podemos reescribir la ecuación (4.77) como sigue:

$$
U(x, y, z)=\frac{m}{2}\left(w_{1}^{2} x^{2}+w_{2}^{2} y^{2}+w_{3}^{2}\left(z+\frac{g}{w_{3}^{2}}\right)^{2}\right)-\frac{1}{2} \frac{m g^{2}}{w_{3}^{2}} .
$$

Los valores propios de energía de una partícula en un potencial dado por la ecuación 4.79 son:

$$
\varepsilon=\hbar w_{1}\left(n_{x}+\frac{1}{2}\right)+\hbar w_{2}\left(n_{y}+\frac{1}{2}\right)+\hbar w_{3}\left(n_{z}+\frac{1}{2}\right)-\frac{1}{2} \frac{m g^{2}}{w_{3}^{2}} ; \quad n_{x}, n_{y}, n_{z} \in \mathbb{N}
$$

La densidad de estados por unidad de energía es:

$$
\Omega(\varepsilon)=\frac{\left(\varepsilon+\frac{m g}{2 w_{3}^{2}}-\frac{\hbar}{2}\left(w_{1}+w_{2}+w_{3}\right)\right)^{2}}{2 \hbar^{3} w_{1} w_{2} w_{3}} .
$$

El número de partículas promedio se define

$$
N=\int_{0}^{\infty} \frac{\Omega(\varepsilon)}{z^{-1} e^{\beta \varepsilon}-1} d \varepsilon+\frac{1}{z^{-1} e^{\beta \varepsilon_{0}}-1},
$$

donde la energía del estado base es:

$$
\varepsilon_{0}=\frac{\hbar}{2}\left(w_{1}+w_{2}+w_{3}\right)-\frac{1}{2} \frac{m g^{2}}{w_{3}^{2}} .
$$

La energía del estado base $\varepsilon_{0}=\hat{\varepsilon}_{0}-\varepsilon_{0}^{(g)}$ la podemos dividir en dos, la energía correspondiente a los osciladores armónicos $\hat{\varepsilon}_{0}$ y la energía debido al campo gravitacional homogéneo $\varepsilon_{0}^{(g)}$. En este caso el número de partículas en el estado se puede escribir como: 


$$
N_{0}=\frac{1}{z^{-1} e^{\beta \varepsilon_{0}}-1} .
$$

Notemos que si $z^{-1} e^{\beta \varepsilon_{0}} \rightarrow 1$ el número de partículas en el estado base $N_{0} \rightarrow \infty$, por lo tanto

$$
z^{-1} e^{\beta \varepsilon_{0}}=e^{\frac{\hat{\varepsilon}_{0}-\varepsilon_{0}^{(g)}-\mu}{\kappa T}} \rightarrow 1
$$

entonces,

$$
\frac{\hat{\varepsilon}_{0}-\varepsilon_{0}^{(g)}-\mu}{\kappa T} \rightarrow 0
$$

observamos que si tomamos un campo gravitacional homogéneo la condensación de Bose-Einstein se verá afectada. El número de partículas promedio al realizar la integral en la ecuación 4.82 da como resultado,

$$
N=\frac{1}{z^{-1} e^{\beta\left(\hat{\varepsilon}_{0}-\varepsilon_{0}^{(g)}\right)}-1}+\frac{(\kappa T)^{3}}{(\hbar) \bar{w}^{3}} g_{3}\left(z e^{-\beta\left(\hat{\varepsilon}_{0}-\varepsilon_{0}^{(g)}\right)}\right),
$$

donde $\bar{w}=w_{1} w_{2} w_{3}$, y $g_{3}\left(z e^{-\beta\left(\hat{\varepsilon}_{0}-\varepsilon_{0}^{(g)}\right)}\right)$ es la función de Bose-Einstein. Si hay condensación tenemos:

$$
N=\left(\frac{\kappa T_{c}}{\hbar \bar{w}}\right)^{3} \zeta(3)
$$

la condición de condensación se obtiene cuando $\mu=\hat{\varepsilon}_{0}-\varepsilon_{0}^{(g)}$ y el potencial químico queda acotado $\mu \epsilon\left(-\infty, \hat{\varepsilon}_{0}-\varepsilon_{0}^{(g)}\right)$.

Ahora vamos a calcular la temperatura de condensación. Para ello vamos a reescribir la función de Bose-Einstein de la siguiente forma:

$$
g_{3}\left(z e^{-\beta \hat{\varepsilon}_{0}}+e^{\varepsilon_{0}^{(g)}}\right)=g_{3}\left(z e^{-\beta \hat{\varepsilon}_{0}}\right)-\frac{m g^{2}}{2 \kappa T w_{3}^{2}} g_{2}\left(z e^{-\beta \hat{\varepsilon}_{0}}\right) .
$$

Sustituyendo la ecuación (4.89) en al ecuación (4.87), cuando $z e^{-\beta \hat{\varepsilon}_{0}} \rightarrow 1$ obtenemos que,

$$
N=\left(\frac{\kappa T_{c}}{\hbar \bar{w}}\right)^{3}\left[\zeta(3)-\frac{m g^{2}}{2 \kappa T_{c} w_{3}^{2}} \zeta(2)\right]
$$


Recordando el número de partículas cuando no hay campo gravitacional $g=0$,

$$
N=\left(\frac{\kappa T_{c}^{o}}{\hbar \bar{w}}\right)^{3} \zeta(3)
$$

igualando las ecuaciones 4.90 y 4.91) y factorizando la función $\zeta(3)$ se obtiene la siguiente expresión

$$
\begin{gathered}
\left(\frac{\kappa T_{c}^{o}}{\hbar \bar{w}}\right)^{3}=\left(\frac{\kappa T_{c}}{\hbar \bar{w}}\right)^{3}\left[1-\frac{m g^{2}}{2 \kappa T_{c} w_{3}^{2}} \frac{\zeta(2)}{\zeta(3)}\right] \\
T_{c}^{o}=T_{c}\left[1-\frac{m g^{2}}{2 \kappa T_{c} w_{3}^{2}} \frac{\zeta(2)}{\zeta(3)}\right]^{1 / 3} .
\end{gathered}
$$

Esta es la temperatura de condensación cuando se tiene un gas confinado por una trampa electromagnética que es modelada con osciladores armónicos anisotrópicos, de hecho así se realiza el experimento, en presencia de un campo gravitacional uniforme. La presencia del campo gravitacional homogéneo $(g \neq 0)$ modifica la temperatura de condensación $\left(T_{c}^{o}<T_{c}\right)$. Observemos que en la ecuación 4.93 solo aparece la frecuencia del oscilador armónico $w_{3}$, esto es debido a que el campo gravitacional solo esta presente en esta dirección. 

CAPÍtulo 5

\section{Conclusiones y Perspectivas}

\subsection{Conclusiones}

En este trabajo se muestra que en la presencia de un campo gravitacional homogéneo las propiedades termodinámicas de un gas ideal de Bose-Einstein se ven modificadas. En particular se analizaron dos casos, primero un gas ideal de BoseEinstein encerrado por un recipiente de volumen $\mathrm{V}$ de altura L con $\mathrm{N}$ partículas indistinguibles que no interactúan entre si, en presencia de un campo gravitacional homogéneo, y segundo un gas ideal de Bose-Einstein confinado por osciladores armónicos anisotrópicos inmerso en un campo gravitacional homogéneo. Cabe señalar que así se hacen los experimentos [70].

El primer caso, que parece ser más un ejercicio de pizarrón que un caso realista, nos ayuda a entender que parámetros termodinámicos se modifican cuando el campo gravitacional esté presente, y que parámetro podría ser el más idóneo para poder medir en el laboratorio. Además, el estudio de un gas bosónico confinado dentro de un recipiente de altura finita en presencia de un campo gravitacional homogéneo es un problema actual, ya que algunos autores consideran que para que 
se presente el fenómeno de condensación debería haber una impureza en la base del contenedor [49], en contraposición con la gran mayoría de trabajos [46, 50]. En este trabajo se muestra que dicha impureza no es necesaria para poder obtener la temperatura de condensación. Desde el punto de vista de la estadística clásica para un gas es bien sabido que en presencia de un campo gravitacional la presión del gas cambia. Para una columna de gas encerrada en un tubo de sección transversal unitaria y sujeta a la acción de un campo gravitacional homogéneo la presión del gas a una altura z está dada por la famosa ecuación de las atmósferas de Perrin $P=P_{0}=e^{-m g z / \kappa T}$. La presión del gas es menor cuando el gas esta sujeto a campo gravitacional homogéneo [64]. Por lo tanto, no nos debe causar sorpresa que la presión obtenida cambie con respecto al caso en el cual no hay campo gravitacional. En el caso de un gas bosónico masivo se tiene que la presión en presencia de un campo gravitacional homogéneo es más grande que la presión de un gas de bosones masivos sin campo gravitacional. La temperatura de condensación para un gas de bosones masivos en presencia de un campo gravitacional homogéneo también se ve modificada y es mayor a la temperatura de condensación cuando el campo gravitacional es nulo.

El segundo caso es un caso más realista, porque el gas no está en un recipiente propiamente dicho, sino se encuentra confinado por trampas electromagnéticas y trampas láseres. Las trampas electromagnéticas se modelan con osciladores armónicos anisotrópicos. Cabe señalar que así se hacen los experimentos en los laboratorios [70]. En este caso también se observa que la presión y la temperatura de condensación cambian cuando se toma en cuenta un campo gravitacional homogéneo. La temperatura de condensación es mayor cuando un campo grav- 
itacional homogéneo esta presente a una temperatura de condensación cuando el campo gravitacional es nulo.

La temperatura de condensación es el parámetro termodinámico más idóneo para verificar el PEE experimentalmente. Una vez que ya hemos fijado nuestro parámetro termodinámico a considerar en los experimentos para poner a prueba el PEE, mencionaremos cual es nuestra propuesta experimental. La propuesta experimental, como ya se mencionó antes, consiste en dejar caer un condensado de Bose-Einstein dentro de la torre de la Universidad de Bremen en Alemania, desde una altura de más de $100 m$, junto con algún dispositivo que nos permita obtener la temperatura de condensación, sin que este altere los resultados del experimento. Cabe mencionar que usualmente la temperatura de condensación de Bose-Einstein se deduce experimentalmente recurriendo a la comparación entre las funciones de distribución de velocidades de Maxwell-Boltzmann y el perfil de densidades del espacio de momentos [46, 71, 72]. Aunque la aproximación parece ser muy satisfactoria, está haciendo uso de la mecánica estadística clásica cuando la temperatura está muy cerca a la temperatura de condensación donde los efectos cuánticos son importantes. Por lo tanto no se pueden despreciar. Como queremos poner a prueba el principio que está detrás de todas las teorías métricas de la gravedad desde el punto de vista meramente cuántico, se necesita una manera alternativa para medir la temperatura de condensación sin recurrir a métodos semi-clásicos para obtenerla. Para evitar problemas de transición cuando hay campo gravitacional y cuando no lo hay (caída libre), el gas ideal de Bose-Einstein (átomos de Rubidio) comenzará a condensar después de que se ha dejado caer el dispositivo. Por lo tanto, la temperatura de condensación que se obtenga es como si no hubiera campo gravitacional. Si PEE es válido se espera que la temperatura de condensación 
obtenida en caída libre sea la misma que se obtiene al realizar el experimento en un laboratorio en ausencia de una campo gravitacional homogéneo. Los resultados obtenidos al realizar el experimento en la torre de Bremen donde el condensado está en caída libre se comparan con los resultados obtenidos en el laboratorio, en donde hay campo gravitacional.

La temperatura es un parámetro termodinámico experimental clásico. Nosotros empleamos a la temperatura de condensación como parámetro experimental para poner a prueba el PEE a nivel cuántico. Para poder usar a la temperatura de condensación como parámetro experimental que ponga a prueba el PEE a nivel cuántico hay que recordar que la temperatura de condensación no debe ser nula $T_{c} \neq 0$, en el caso de tener una temperatura de condensación nula $T_{c}=0$ se estaría recurriendo a la estadística de Maxwell-Boltzmann y esta es un estadística clásica y no serviría para poner a prueba el PEE a nivel cuántico. Por lo tanto, al usar la estadística de Bose-Einstein, que es una estadística cuántica, y requerir una temperatura de condensación que no sea nula, podemos poner a prueba el PEE a nivel cuántico. La temperatura de condensación es un parámetro termodinámico que pone a prueba el PEE a nivel cuántico y no el PED. Para que el gas se encuentre dentro de una fase condensada la temperatura solo puede tomar los valores $0<T<T_{c}$, por arriba de la temperatura critica no habrá fase condensada, de hecho para temperaturas muy alejadas de la temperatura critica $T \gg T_{c}$, el gas se comporta como una gas clásico. Además, el PED está asociado con la idea de trayectoria y en mecánica cuántica este concepto no es válido. PEE exige la validez del PED y el cumplimiento de la ILL y ILP. Por lo tanto la propuesta experimental intenta probar el PEE y no el PED. El PEE nos dice, en pocas palabras, que un experimento no-gravitacional que se realice en caída libre es igual a 
un experimento no-gravitacional sin campo gravitacional, y por lo tanto es válida la Relatividad Especial. Las limitaciones del experimento provienen del cumplimiento del teorema de planitud, el cual nos dice que hay una vecindad alrededor de un punto $\mathrm{P}$ en el cual la métrica está dada por la métrica de Minkowski $\eta_{\alpha \beta}$. En dicha vecindad el campo gravitacional es nulo. Por lo tanto si queremos que nuestro experimento en caída libre sea válido, tiene que cumplir con el teorema de planitud [73. Recordemos el límite de campo gravitacional débil $g_{\alpha \beta}=\eta_{\alpha \beta}+h_{\alpha \beta}$ donde $h \ll 1$. Para cumplir con este límite hay que recordar que no todas las segundas derivadas de la métrica son cero, entonces, alrededor de un punto con coordenadas $x_{0}^{\mu^{\prime}}$ se tiene que cumplir que $\left|\frac{1}{2}\left(x^{\gamma^{\prime}}-x_{0}^{\gamma^{\prime}}\right)\left(x^{\lambda^{\prime}}-x_{0}^{\lambda^{\prime}}\right) \frac{\partial^{2} g_{\alpha \beta}\left(x_{0}^{\mu^{\prime}}\right)}{\partial x^{\lambda^{\prime}} x^{\gamma^{\prime}}}\right| \ll 1$. Esta cantidad es aproximadamente $1 \times 10^{-5}<1$, para una altura de $100 \mathrm{~m}$ sobre la superficie de la tierra, lo que muestra que el teorema de planitud se cumple para este experimento.

La factibilidad de realizar este experimento la describiré en la siguiente tabla para los dos casos en que tenemos un gas de Bose-Einstein en presencia de un campo gravitacional homogéneo encerrado por un recipiente y un gas de Bose-Einstein confinado por trampas electromagnéticas modeladas por osciladores armónicos anisotrópicos, se tomarán los resultados experimentales para el Rubidio [70], Sodio [71] y Litio [74],

\begin{tabular}{|c|c|c|}
\hline Elemento & Gas encerrado en un recipiente & Gas confinado por osciladores armónicos \\
& $\frac{T_{c}-T_{c}^{0}}{T_{c}^{0}}$ & anisotrópicos \\
& 4.57407 & $\frac{T_{c}-T_{c}^{0}}{T_{c}^{0}}$ \\
\hline${ }^{87} \mathrm{Rb}$ & 8.26362 & 0.000172 \\
${ }^{23} \mathrm{Na}$ & 28.3967 & 0.007556 \\
${ }^{7} \mathrm{Li}$ & & 0.017047 \\
\hline
\end{tabular}


En esta tabla, $T_{c}$ es la temperatura de condensación en presencia de un campo gravitacional uniforme, según sea el caso; recipiente de volumen finito o trampa electromagnética modelada con osciladores armónicos anisotrópicos, donde se consideró $g=9.8 \frac{m}{s^{2}}$ y $T_{c}^{0}$ es la temperatura de condensación que se obtiene al realizar el experimento. El modelo de pizarrón no es un buen modelo, aunque la factibilidad de lograr el experimento sea mayor a la unidad, es un caso ideal debido que el experimento se realiza encerrando el gas en un recipiente. Pero este modelo nos ayuda a darnos cuenta como se ven modificadas las propiedades termodinámicas del gas de Bose-Einstein masivo en presencia de un campo externo, en este caso un campo gravitacional homogéneo, y nos da la pauta para elegir a la temperatura de condensación como nuestro parámetro experimental a considerar para verificar el PEE. La factibilidad de realizar el experimento con una gas de Bose-Einstein masivo confinado por medio de osciladores anisotrópicos depende de tres cantidades físicas principalmente, la masa del gas, la densidad del gas, y las frecuencias de atrapamiento. Como se puede observar, según la tabla, el isótopo del Litio-7 es el gas más idóneo para poner a prueba el PEE, pero recordemos que la temperatura de condensación que estamos usando aquí es la obtenida experimentalmente, donde los experimentales hacen alusión a métodos semi-clásicos para obtenerla. Por está razón no hay que descartar la posibilidad de usar cualquiera de los otros gases en un experimento donde la temperatura de condensación no se obtenga a partir de métodos semi-clásicos. 


\subsection{Perspectivas}

Nuestro modelo está diseñado para gases altamente diluidos, las partículas que constituyen al gas se han considerado que no interactúan entre si, además el acoplamiento del spin con momento magnético no se ha considerado. Lo que sigue es estudiar sistemas con estas características, en otras palabras, estudiar un gas de Bose-Einstein donde la interacción entre las partículas no sea despreciable, inmerso en un campo gravitacional homogéneo donde también se considere el acoplamiento del spin con el momento magnético, estudiar como se modifican las

propiedades termodinámicas al considerar que el gas se encuentra confinado por medio de osciladores armónicos anisotrópicos rotantes. También se estudiarán otras propiedades termodinámicas como la energía interna del gas y el calor específico. El calor específico es una cantidad termodinámica esencial en el estudio de transiciones de fase. Se observará como se ve modificado el calor específico en presencia de un campo gravitacional homogéneo y como esto nos puede ayudar a poner a prueba el PEE. Además, se estudiará como la presencia de una campo gravitacional homogéneo modifica el numero de partículas en el estado base y la energía interna de un gas de Bose-Einstein masivo como función de la temperatura. Asimismo, se estudiará que tan viable es poner a prueba el PEE al considerar el tamaño del condensado (el volumen que ocupa el condensado no es un volumen físico es generado por las trampas electromagnéticas que se modelan con osciladores armónicos anisotrópicos, a esto se le conoce como el tamaño del condensado) cuando este se encuentre en presencia de un campo gravitacional homogéneo con respecto al caso cuando no hay campo gravitacional. 



\section{Bibliografía}

1. C. M. Will, Theory and Experiment in Gravitational Physics, Cambridge University Press, Cambridge, (1993).

2. G. Galilei, Discorsi e dimostriazioni matematiche intorno a due nuove scienze, Elzevir, Leiden (1683). English Translation by H. Crew and A. de Salvio, Dialogues Concerning Two New Sciences, Macmillan, New York, (1914); reprinted by Dover, New York, (1954).

3. M. P. Haugan and C. Lämmerzahl, Principles of Equivalence: Their Role in Gravitation Physics and Experiments That Test Them, in Gyros, Clocks, Interferometers...; Testing Relativistic Gravity in Space, C. L“ammerzahl, C. W. F. Everitt, and F. W. Hehl, editors, (Springer-Verlag, Heidelberg, 2001).

4. J. J. Sakurai, Modern Quantum Mechanics, Addison-Wesley (1994).

5. H. Dittus and C. Lämmerzahl, Experimental Test of the Equivalence Principle and Newton's Law in Space, GRAVITATION AND COSMOLOGY: 2nd Mexican Meeting on Mathematical and Experimental Physics. AIP Conference Proceedings, Volume 758, pp. 95-112 (2005).

6. I. Newton, Philosophiae Naturalis Principia Mathematica (London 1686).

7. F. W. Bessel, Pogg. Ann. 25, 401 (1832).

8. H. H. Potter, Proc. Roy. Soc. 104, 588 (1923).

9. R. V. Eötvös, Math. u. Naturw. Ber. aus. Ungarn 8, 65 (1890).

10. R. V. Eötvös, V. Pekar, and E. Fekete, Beiträge zum Gesetze der Proportionalität von Trägheit und Gravität, Ann. Phys. (Leipzig), 68, 11-66, (1922). 
11. G. L. Stubbs, E. A. Adelberger, F. J. Raab, J. H. Gundlach, B. R. Heckel, K. D. McMurry, H. E. Swanson, and R. Wtanabe, Search for an intermediate-range interaction, Phys. Rev. Lett., 58:1070, 1987.

12. G. L. Smith, C. D. Hoyle, J. H. Gundlach, E. G. Adelberger, B. R. Heckel, and H. E. Swanson, Short range test of the equivalence principle, Phys. Rev., D 61:022001, 2000.

13. Y. Su, B. R. Heckel, E. G. Adelberger, J. H. Gundlach, M. Harris, G. L. Smith, and H. E. Swanson, New test of the universality of free fall, Phys. Rev., D 50:3614, 1994.

14. P. G. Roll, R. Krotkov, R. H. Dicke, Ann. Phys. (N.Y.), 26:442, 1967.

15. V. Braginsky, V. Panov, Sov. Phys. JETP, 34:463, 1972.

16. Nick Lockerbie, John C. Mester, Rodney Torii, Stefano Vitale, Paul W. Worden STEP: A Status Report, C. Lämmerzahl, C.W.F. Francis, and F.W. Hehl (Eds.): LNP 562, pp. 213-247, 2001. Springer-Verlag Berlin Heidelberg 2001.

17. Clifford M. Will, The Confrontation between General Relativity and Experiment, Living Rev. Relativity $9,(2006)$.

18. A. A. Michelson, and E. W. Morley, On the Relative Motion of the Earth and the Luminiferous Ether, Am. J. Sci., 34, 333-345, (1887). Related online version: http://www.aip.org/history/gap/Michelson/Michelson.html.

19. R. S. Shankland, S. W. McCuskey, F. C. Leone, and G. Kuerti,New analysis of the interferometer observations of Dayton C. Miller, Rev. Mod. Phys., 27, 167-178, (1955).

20. T. S. Jaseja, A. Javan, J. Murray, and C.H. Townes, Test of special relativity or of the isotropy of space by use of infrared masers, Phys. Rev., 133, A1221-A1225, (1964).

21. A. Brillet, and J. L. Hall, Improved laser test of the isotropy of space, Phys. Rev. Lett., 42, 549-552, (1979).

22. H. E. Ives, and G. R. Stilwell, An experimental study of the rate of a moving atomic clock, J. Opt. Soc. Am., 28, 215-226, (1938).

23. B. Rossi, and D. B. Hall, Variation of the rate of decay of mesotrons with momentum, Phys. Rev., 59, 223-228, (1941).

24. F. J. M. Farley, J. Bailey, R. C. A. Brown, M. Giesch, H.Jöstlein, S. van der Meer, E. Picasso, and M. Tannenbaum, The Anomalous Magnetic Moment of the Negative Muon, Nuovo Cimento, 45, 281-286, (1966).

25. K. Brecher, Is the speed of light independent of the velocity of the source?, Phys. Rev. Lett., 39, 1051-1054, (1977). 
26. T. Alväger, F. J. M. Farley, J. Kjellman, and I. Wallin, Test of the second postulate of special relativity in the GeV region, Phys. Lett., 12, 260-262, (1977).

27. D. C. Champeney, G. R. Isaak, and A. M. Khan, An "aether drift" experiment based on the Mössbauer effect, Phys. Lett., 7, 241-243, (1963).

28. E. Riis, L. U. A. Anderson, N. Bjerre, O. Poulson, S. A. Lee, and J. L. Hall, "Test of the Isotropy of the Speed of Light Using Fast-Beam Laser Spectroscopy, Phys. Rev. Lett., 60, 81-84, (1988).

29. T. P. Krisher, L. Maleki, G. F. Lutes, L. E. Primas, R. T. Logan, J. D. Anderson, and C. M. Will, Test of the isotropy of the one-way speed of light using hydrogen-maser frequency standards, Phys. Rev. D, 42, 731-734, (1990).

30. P. A. M. Dirac, The Quantum Theory of Electron, Proc. R. Soc. London, A 117, 610-624 (1928).

31. C. H. Lineweaver, L. Tenorio, G. F. Smoot, P. Keegstra, A. J. Banday, and P. Lubin, The dipole observed in the COBE DMR 4 year data, Astrophys. J., 470, 38-42, (1996).

32. V. W. Hughes, H. G. Robinson, and V. Beltran-Lopez, Upper limit for the anisotropy of inertial mass from nuclear resonance experiments, Phys. Rev. Lett., 4, 342-344, (1960).

33. R. W. P. Drever, A search for anisotropy of inertial mass using a free precession technique, Philos. Mag., 6, 683-687, (1961).

34. J. D. Prestage, J. J. Bollinger, W. M. Itano, and D. J. Wineland, Limits for Spatial Anisotropy by Use of Nuclear-Sspin-Polarized 9Be+ Ions, Phys. Rev. Lett., 54, 2387-2390, (1985).

35. S. K. Lamoreaux, J. P. Jacobs, B. R. Heckel, F. J. Raab, and E. N. Fortson, New limits on spatial anisotropy from optically-pumped $201 \mathrm{Hg}$ and 199Hg, Phys. Rev. Lett., 57, 3125-3128, (1986).

36. T. E. Chupp, R J. Hoare, R. A. Loveman, E. R. Oteiza, J. M. Richardson, M. E. Wagshul, and A. K. Thompson, Results of a new test of local Lorentz invariance: A search for mass anisotropy in 21Ne, Phys. Rev. Lett., 63, 1541-1545, (1989).

37. M. P. Haugan, and C. M. Will, Modern tests of special relativity, Phys. Today, 40, 69-76, (1987).

38. P. Wolf, S. Bize, A. Clairon, A. N. Luiten, G. Santarelli, and M. E. Tobar, Tests of Lorentz invariance using a microwave resonator, Phys. Rev. Lett., 90, 060402-1-4, (2003).

39. J. A. Lipa, J. A. Nissen, S. Wang, D. A. Stricker, and D. Avaloff, New limit on signals of Lorentz violation in electrodynamics, Phys. Rev. Lett., 90, 060403-1-4, (2003).

40. H. Müller, S. Herrmann, C. Braxmaier, S. Schiller, and A. Peters, Modern Michelson-Morley experiment using cryogenic optical resonators, Phys. Rev. Lett., 91, 020401-1-4, (2003).

41. P. Antonini, M. Okhapkin, E. Goklu, and S. Schiller Test of constancy of speed of light with rotating cryogenic optical resonators, Phys. Rev. A, 71, 050101, (2005). 
42. P. L. Stanwix, M. E. Tobar, P. Wolf, M. Susli, C. R. Locke, E. N. Ivanov, J. Winterflood, and F. van Kann, Test of Lorentz Invariance in Electrodynamics Using Rotating Cryogenic Sapphire Microwave Oscillators, Phys. Rev. Lett., 95(4), 040404, (jul, 2005).

43. M. P. Haugan, and C. L. Lämmerzahl, On the interpretation of Michelson-Morley experiments, Phys. Lett. A, 282, 223-229, (2001).

44. R. V. Pound and G. A. Rebka, Apparent weight of photons, Phys. Rev. Lett., 4, 337-41 (1960).

45. R. V. Pound, G. A. Rebka and J. L. Snider Effect of gravity on gamma radiation, Phys. Rev., 140, B788-803.

46. R. K. Pathria, Statistical Mechanics, Butterworth Heineman, Oxford (1996).

47. Greiner, Neise Stocker, Thermodynamics and Statistical Mechanics, Springer-Verlag, New York, (1995).

48. Leopoldo Garcia-Colín S., Francisco Ramos Gómez, Raúl Rechtman,Temas Selectos de Mecánica Estadística, El Colegio Nacional, México(1992).

49. R. M. Cavalcanti, P. Giacconi, G. Pupillo, R. Soldati, Bose-Einstein Condensation in the Presence of a Uniform Field and Point-Like Impurity, PhysRevA., 65.053606.

50. R. Eisenschitz, Statistical theory of irreversible processes, Oxford University Press, (1958).

51. A. J. Leggett, Bose-Einstein condensation in the alkali gases: Some fundamental concepts, Rev. Mod. Phys. 73, 307 (2001).

52. K. L. Corwin, S. J. M. Kuppens, D. Cho, and C. E. Wieman, Spin-Polarized Atoms in a Circularly Polarized Optical Dipole Trap, Phys. Rev. Lett. 83, 1311, (1999).

53. J. Stenger, D. M. Stamper-Kurn, M. R. Andrews, A. P. Chikkatur, S. Inouye, H-J. Miesner, and W. Ketterle, Optically confined Bose-Einstein condensates, J. Low Temp. Phys. 113, 167, (1998).

54. W. Petrich, M. H. Anderson, J. R. Ensher, and E. A. Cornell, Stable, Tightly Confining Magnetic Trap for Evaporative Cooling of Neutral Atoms, Phys. Rev. Lett. 74, 3352, (1995).

55. D. E. Pritchard, Cooling Neutral Atoms in a Magnetic Trap for Precision Spectroscopy, Phys. Rev. Lett. 51, 1336, (1983),

56. J. L. Anderson, Principle of relativity physics, New York: Academic Press, pp. 329-343, (1967).

57. A. Trautman, The General theory of Relativity, Sov. Phys. Usp., 9 319, (1966).

58. C. Brans, and R. H Dicke, Mach's principle and a relativistic theory of gravitation, Phys. Rev. 124, pp. 925-935, (1961).

59. N. Rosen, A bi-metric theory of gravitation, J. Gen. Rel. and Grav. 4, pp. 435-447, (1973).

60. F. Belinfante, and J. Swihart. Phenomenological Linear Theory of Gravitation. I. Classical Mechanics, Annals of Physics 1, pp. 168-195, (1957). 
61. F. Belinfante, and J. Swihart. Phenomenological Linear Theory of Gravitation. II. Interaction with Maxwell Field, Annals of Physics 1, pp. 196-212 (1957).

62. F. Belinfante, and J. Swihart. Phenomenological Linear Theory of Gravitation. III. Interaction with Spinning Electron, Annals of Physics 2, pp. 81-99 (1957).

63. L. P. Eisenhart, An Introduction To Differential Geometry - With The Use Of Tensor Calculus, Princeton University Press: Oxford University Press, (1940).

64. Leopoldo Garcia-Colín Scherer, Introducción a la Física Estadística, El Colegio Nacional, México (2005).

65. F. London, On the Bose-Einstein Condensation, Phys. Rev. 54, pp. 947-954, (1938).

66. T. Könemann, W. Brinkmann, E. Göklü, C. Lämmerzahl, H. Dittus, T. van Zoest, E. Rasel, W. Ertmer, W. Lewoczko-Adamczyk, M. Schiemanck, A. Peters, A. Vogel, G. Johannsen, S. Widlfang, K. Bongs, K. Sengstock, E. Kajari, G. Nandi, W. P. Schleich, R. Walser, A freely falling magnetooptical trap drop tower experiment, Applied Physics B 89, 431 (2007).

67. S. Flügge Practical Quantum Mechanics, Springer-Verlag, New York, (1994)

68. R. Shankar, Principles of Quantum Mechanics, New York : Plenum, (1994).

69. A. Messiah, Mecánica Cuántica, Tomo 1, Editorial Tecnos (1983).

70. M. H. Anderson, J. R. Ensher, M. R. Matthews, C. E. Wieman, and E. A. Cornell, Observation of Bose-Einstein condensation in a dilute atomic vapor, Science 269, 198-201 (1995).

71. K. B. Davis, M. O. Mewes, M. R. Andrews, N. J. van Druten, D. S. Durfee, D. M. Kurn, and W. Ketterle, Bose-Einstein condensation in a gas of sodium atoms Phys. Rev. Lett. 75, 3969-3973 (1995).

72. M. R. Andrews, M. O. Mewes, N. J. van Druten, D. S. Durfee, D. M. Kurn, and W. Ketterle, Direct, Non-desructive Observation of a Bose Condensate, Science 75, 84-87 (1996).

73. B. F. Schutz, A first course in general relativity, Cambridge University, (1990).

74. C. C. Bradley, C. A. Sackett, J. J. Tollet, and R. G. Hulet, Evidence of Bose-Einstein condensation in an atomic gas with attractive interactions, Phys. Rev. Lett. 75, 1687-1690 (1995).

75. R. G. Newton, Scattering Theory of Waves and Particles, 2nd. ed. New York: McGraw-Hill, pp. 233, (1982). 

ApÉNDICE A

\section{Apéndice A: Teoremas}

\section{A.1. Teoremas}

\section{A.1.1. Teorema de Convergencia Absoluta}

Primer Teorema: Convergencia absoluta

Una serie $\sum_{n=1}^{\infty} a_{n}$ es convergente si la serie(términos positivos) $\sum_{n=1}^{\infty}\left|a_{n}\right|$ converge y si $\sum_{n=1}^{\infty} a_{n}=s, \sum_{n=1}^{\infty}\left|a_{n}\right|=S \Rightarrow|s| \leq|S|$.

\section{A.1.2. Teorema de sucesiones crecientes}

Segundo Teorema: Si $\sum_{l=0}^{k} a_{l}=b_{k}$ es creciente tal que $b_{k} \leqslant c_{k}$ donde $c_{k} \rightarrow c$ si $k \rightarrow \infty \quad \therefore b_{k}$ también converge.

\section{Dem.}

Sea $\varepsilon>0$ por definición $b=\inf \left\{\right.$ cotas superiores de $\left.\left\{b_{k}\right\}\right\}$

$\forall \varepsilon>0, \exists \tilde{k} \in \mathbb{N}$ tal que $b-\varepsilon<b_{\tilde{k}}$

$\Rightarrow \varepsilon>0, \quad \exists \tilde{k} \in \mathbb{N}$ tal que $\quad 0 \leq b-b_{\tilde{k}}<\varepsilon$ 


$$
\begin{aligned}
& \text { como } \quad b_{\tilde{k}} \leq b_{l}, \quad l \geq \tilde{k} \text { (es creciente) } \\
& (-1)\left(b_{\tilde{k}} \leq b_{l}\right) \quad \Rightarrow \quad-b_{\tilde{k}} \geq-b_{l} \\
& -b_{\tilde{k}}+b \geq-b_{l}+b(\text { tomo el valor absoluto) } \\
& \left|b-b_{\tilde{k}}\right| \geq\left|b-b_{b}\right| \quad \Rightarrow\left|b_{\tilde{k}}-b\right| \geq\left|b_{l}-b\right| \\
& \therefore\left|b_{l}-b\right| \leq\left|b_{\tilde{k}}-b\right|<\varepsilon, \quad \forall l \geq \tilde{k} .
\end{aligned}
$$


ApÉNDICE B

\section{Apéndice B: Teoría de Perturbaciones independiente del tiempo}

\section{B.1. Teoría de Perturbaciones independiente del tiempo}

El método de aproximación que utilizaremos aquí es una teoría de perturbaciones independiente del tiempo, también se le conoce como la teoría de perturbaciones de Rayleigh-Schrödinger. Consideraremos un Hamiltoniano $H$ de tal forma que se pueda escribir en dos partes, es decir:

$$
H=H_{0}+V .
$$

Cuando $V=0$ los vectores propios de energía exactos $\left|n^{(0)}\right\rangle$ y los valores propios de energía $E_{n}^{(0)}$ exactos son conocidos y corresponden al Hamiltoniano sin perturbar.

$$
H_{0}\left|n^{(0)}\right\rangle=E_{n}^{(0)}\left|n^{(0)}\right\rangle \text {. }
$$

Requerimos encontrar los vectores propios y valores propios aproximados para el problema del Hamiltoniano completo

$$
\left(H_{0}+V\right)|n\rangle+E_{n}|n\rangle
$$


donde $V$ es conocido como la perturbación; no es, en general, el operador del potencial completo. Por ejemplo, supongamos que consideramos el átomo de hidrógeno en un campo eléctrico o magnético externo. El Hamiltoniano sin perturbar $H_{0}$ se ha tomado ser la energía cinética $\frac{\mathbf{p}^{2}}{2 m}$ y el potencial de Coulomb debido a la presencia del núcleo del protón $\frac{-e^{2}}{r}$. Solo la parte del potencial debido a la interacción con el campo externo $\mathbf{E}$ o $\mathbf{B}$ es representado por la perturbación $V$. En vez de trabajar con la ecuación B.3 se acostumbra resolver

$$
\left(H_{0}+\lambda V\right)|n\rangle+E_{n}|n\rangle
$$

donde $\lambda$ es un parámetro real continuo. Este parámetro es introducido para observar el número de veces que la perturbación entra. Al final del cálculo podemos poner $\lambda \rightarrow 1$ para regresar al caso (B.3). El parámetro $\lambda$ puede ser visualizado para variar continuamente desde 0 a 1 . El caso $\lambda=0$ corresponde al problema sin perturbar y $\lambda=1$ corresponde al problema completamente perturbado (B.3). En situaciones físicas donde este método de aproximación es aplicable, esperamos ver una transición del estado $\left|n^{0}\right\rangle$ al estado $|n\rangle$ y de $E_{n}^{(0)}$ al $E_{n}$ cuando $\lambda$ cambié su valor de 0 a 1.

El resto del método, es sobre el desarrollo de los valores propios de energía y vectores propios en potencias de $\lambda$. Esto significa que implícitamente asumiremos la analicidad de los valores propios de la energía y vectores propios en un plano complejo $\lambda$ alrededor de $\lambda=0$. Una buena aproximación puede ser obtenida al tomar solo uno o dos términos en el desarrollo. 


\section{B.1.1. El Problema de dos estados}

Veremos como el desarrollo en $\lambda$ en efecto podría ser válido en el problema de dos estados que hemos encontrado muchas veces. Tenemos un Hamiltoniano que puede ser escrito como

$$
\begin{aligned}
H & =E_{1}^{(0)}\left|1^{(0)}\right\rangle\left\langle 1^{(0)}\left|+E_{2}^{(0)}\right| 2^{(0)}\right\rangle\left\langle 2^{(0)}\right| \\
& +\lambda V_{12}\left|1^{(0)}\right\rangle\left\langle 2^{(0)}\left|+\lambda V_{21}\right| 2^{(0)}\right\rangle\left\langle 1^{0}\right|,
\end{aligned}
$$

donde $\left|1^{(0)}\right\rangle$ y $\left|2^{(0)}\right\rangle$ son los vectores propios de energía para el problema con $\lambda=0$, y consideramos el caso $V_{11}=V_{22}=0$. En esta representación el Hamiltoniano $H$ puede ser representado por una matriz de dos por dos de la siguiente forma:

$$
\mathbf{H}=\left(\begin{array}{ll}
E_{1}^{(0)} & \lambda V_{12} \\
\lambda V_{21} & E_{2}^{(0)}
\end{array}\right),
$$

donde hemos usado la base formada por los vectores propios de energía sin perturbar. La matriz $V$ debe, de hecho, ser Hermitiano; resolveremos el caso cuando $V_{12}$ y $V_{21}$ son reales:

$$
V_{12}=V_{12}^{*}, \quad V_{21}=V_{21}^{*}
$$

desde ahora, por ser Hermitianos

$$
V_{21}=V_{12}
$$

Estos siempre puede ser hecho al ajustar la fase de $\left|2^{(0)}\right\rangle$ relativa a esta de $\left|1^{(0)}\right\rangle$. El problema de obtener los valores propios de energía aquí es completamente análogo al resolver el problema de la orientación del spin, donde el análogo de la ecuación B.1.1) es 


$$
\mathbf{H}=a_{0}+\sigma \cdot \mathbf{a}=\left(\begin{array}{cc}
E_{1}^{(0)} & \lambda V_{12} \\
\lambda V_{21} & E_{2}^{(0)}
\end{array}\right),
$$

donde asumimos $\mathbf{a}=\left(a_{1}, 0, a_{3}\right)$ sea pequeña y $a_{0}, a_{1}, a_{3}$ son todos reales.Los valores propios para este problema son conocidos ser solo:

$$
E=a_{0} \pm \sqrt{a_{1}^{2}+a_{3}^{2}} .
$$

Por analogía a los correspondientes valores propios para son:

$$
\left\{\begin{array}{l}
E_{1} \\
E_{2}
\end{array}\right\}=\frac{\left(E_{1}^{0}+E_{2}^{0}\right)}{2} \pm \sqrt{\left[\frac{\left(E_{1}^{0}-E_{2}^{0}\right)^{2}}{4}+\lambda^{2}\left|V_{12}\right|^{2}\right]} .
$$

Supongamos $\lambda\left|V_{12}\right|$ es pequeña comparada con la escala de energía relevante, la diferencia de los valores propios de energía del problema sin perturbar:

$$
\lambda\left|V_{12}\right| \ll\left|E_{1}^{(0)}-E_{2}^{(0)}\right| .
$$

Podemos entonces usar

$$
\sqrt{1+\epsilon}=1+\frac{1}{2} \epsilon-\frac{1}{8} \epsilon^{2}+\cdots
$$

Para obtener el desarrollo de los valores propios de energía en la presencia de la perturbación $\lambda\left|V_{12}\right|$, es decir,

$$
\begin{aligned}
& E_{1}=E_{1}^{(0)}+\frac{\lambda^{2}\left|V_{12}\right|^{2}}{\left(E_{1}^{(0)}-E_{2}^{(0)}\right)}+\cdots \\
& E_{2}=E_{2}^{(0)}+\frac{\lambda^{2}\left|V_{12}\right|^{2}}{\left(E_{2}^{(0)}-E_{1}^{(0)}\right)}+\cdots
\end{aligned}
$$

Estas son las expresiones que podemos fácilmente obtener usando el formalismo general. Como un ejemplo, consideremos un problema de una dimensión que envuelve una partícula de masa m en un potencial cuadrado de profundidad $V_{0}$ 


$$
V(\mathbf{x})= \begin{cases}-V_{0}, & \text { para }-\mathbf{a}<\mathbf{x}<\mathbf{a} \\ 0, & \text { para }|x|>a\end{cases}
$$

Este problema admite un estado límite de energía,

$$
E=-|\lambda V|^{2}, \quad \lambda>0 \text { para la atraccion. }
$$

Consideramos el pozo cuadrado como una perturbación muy débil que será agregado al Hamiltoniano de partícula libre y interpretaremos el resultado de la ecuación B.13 como el desplazamiento de la energía en el estado base desde cero a $|\lambda V|^{2}$. Especialmente, porque la ecuación (B.13) es cuadrático en $V$, podemos estar tentados a asociar esto como la energía de desplazamiento del estado base calculado de acuerdo a la teoría de perturbaciones de segundo orden. Sin embargo, este punto de vista es falso porque si este fuera el caso, el sistema podría también admitir un estado $E<0$ para un caso de potencial repulsivo con $\lambda$ negativa, lo cual seria un claro disparate.

Ahora examinaremos el radio de convergencia del desarrollo en serie de (B.11B.12. Si regresamos a la expresión exacta de la ecuación (B.1.1) y consideramos esta ecuación como una función de un variable compleja $\lambda$, observaremos como $|\lambda|$ va creciendo desde cero, los puntos desviados están en

$$
\lambda\left|V_{12}\right|=\frac{ \pm \imath\left(E_{1}^{(0)}-E_{2}^{(0)}\right)}{2}
$$

La condición para la convergencia del desarrollo en serie para $\lambda=1$ el caso completamente perturbado es

$$
\left|V_{12}\right|<\frac{\left|E_{1}^{(0)}-E_{2}^{(0)}\right|}{2} .
$$


Si esta condición no se cumple, el desarrollo en teoría de perturbaciones de las ecuaciones B.11 B.12 no tiene sentido. 
ApÉndice $\mathrm{C}$

\section{Apéndice C: Convergencia de Series}

demostraremos que estas dos series convergen

$$
\begin{aligned}
& \sum_{q=0}^{\infty} \frac{1}{(2 q+1)^{5}[2 n-(2 q+1)]^{5}}, \\
& \sum_{q=0}^{\infty} \frac{1}{(2 q+1)^{4}[2 n-(2 q+1)]^{4}} .
\end{aligned}
$$

Se desarrollará primero la serie C.1

$$
\begin{aligned}
\sum_{q=0}^{\infty} \frac{1}{(2 q+1)^{5}[2 n-(2 q+1)]^{5}} & =\sum_{q=0}^{n-1} \frac{1}{(2 q+1)^{5}[2 n-(2 q+1)]^{5}} \\
& +\sum_{q=n}^{\infty} \frac{1}{(2 q+1)^{5}[2 n-(2 q+1)]^{5}} .
\end{aligned}
$$

Hasta aquí no se ha realizado ninguna aproximación.

Primera aproximación.

$$
\sum_{q=0}^{n-1} \frac{1}{(2 q+1)^{5}[2 n-(2 q+1)]^{5}} \quad \forall q=0,2, \ldots, n-1 \quad \Rightarrow 2 n>2 q+1 .
$$

La primera aproximación que se va usar es la siguiente 


$$
\begin{aligned}
{[2 n-(2 q+1)]^{5} \approx(2 n)^{5} \therefore 2 n>2 q+1 } & \forall q=0,2, \ldots, n-1, \\
\sum_{q=0}^{n-1} \frac{1}{(2 q+1)^{5}[2 n-(2 q+1)]^{5}} & \approx \sum_{q=0}^{n-1} \frac{1}{(2 q+1)^{5}} \frac{1}{(2 n)^{5}} \\
& =\frac{1}{32 n^{5}} \sum_{q=0}^{n-1} \frac{1}{(2 q+1)^{5}} .
\end{aligned}
$$

Recordando: Si a,b,c $\in \mathbb{N}^{+}$y a,b,c $\neq 0$, donde $b=a c$

$$
\left|\frac{1}{a}\right| \geq\left|\frac{1}{b}\right| \Rightarrow\left|\frac{1}{a}\right| \geq\left|\frac{1}{a c}\right|=\left|\frac{1}{a}\right|\left|\frac{1}{c}\right| .
$$

Utilizando la ultima propiedad en la ecuación (C.6) se obtiene:

$$
\frac{1}{(2 n)^{5}}\left|\frac{1}{(2 q+1)^{5}}\right| \geq\left|\frac{1}{(2 q+1)^{5}} \frac{1}{[2 n-(2 q+1)]^{5}}\right|=\left|\frac{1}{(2 q+1)^{5}} \frac{-1}{[(2 q+1)-n]^{5}}\right|
$$

Por lo tanto

$$
\frac{1}{(2 n)^{5}} \sum_{q=0}^{\infty}\left|\frac{1}{(2 q+1)^{5}}\right| \geq \sum_{q=0}^{\infty}\left|\frac{1}{(2 q+1)^{5}} \frac{1}{[(2 q+1)-n]^{5}}\right| .
$$

Bastará con demostrar que la siguiente serie converge

$$
\sum_{q=0}^{\infty}\left|\frac{1}{(2 q+1)^{5}}\right|
$$

Si la serie C.10 converge, entonces la siguiente serie también será convergente

$$
\sum_{q=0}^{\infty}\left|\frac{1}{(2 q-1)^{5}} \frac{1}{[(2 q-1)-n]^{5}}\right|
$$

Pero lo que nosotros obtuvimos es:

$$
\sum_{q=0}^{n-1} \frac{1}{(2 q+1)^{5}}
$$


Para demostrar que esta serie converge se utilizarán dos teoremas.

Primer Teorema: Convergencia absoluta

Una serie $\sum_{n=1}^{\infty} a_{n}$ es convergente si la serie(términos positivos) $\sum_{n=1}^{\infty}\left|a_{n}\right|$ converge y si $\sum_{n=1}^{\infty} a_{n}=s, \sum_{n=1}^{\infty}\left|a_{n}\right|=S \Rightarrow|s| \leq|S|$. Utilizando el primer teorema se obtiene:

$$
\sum_{q=0}^{\infty} \frac{1}{(2 q+1)^{5}}
$$

es convergente si esta ecuación converge

$$
\sum_{q=0}^{\infty}\left|\frac{1}{(2 q+1)^{5}}\right|
$$

\section{Segundo Teorema:}

Si $\sum_{l=0}^{k} a_{l}=b_{k}$ es creciente tal que $b_{k} \leqslant c_{k}$ donde $c_{k} \rightarrow c$ si $k \rightarrow \infty \quad \therefore b_{k}$ también converge.

Utilizando estos dos teoremas se obtiene:

$$
\begin{gathered}
\sum_{q=0}^{\infty} \frac{1}{(2 q+1)^{5}} \leq \sum_{q=0}^{\infty} \frac{1}{(q)^{5}}, \\
\therefore \quad \sum_{q=0}^{\infty} \frac{1}{(2 q+1)^{5}} \quad \text { esta serie converge. }
\end{gathered}
$$

Usando el segundo teorema se observa que esta serie también converge

$$
\sum_{q=0}^{n-1} \frac{1}{(2 q+1)^{5}}
$$

Como se mencionó antes, para que esta serie sea convergente bastará con demostrar que la serie (C.17) converge. Debido a que la serie C.17) converge, entonces esta serie también converge. 


$$
\sum_{q=0}^{\infty} \frac{1}{(2 q+1)^{5}} \frac{1}{[(2 q+1)-n]^{5}}
$$

De la misma forma se demuestra para esta serie su convergencia

$$
\sum_{q=0}^{\infty} \frac{1}{(2 q+1)^{4}} \frac{1}{[(2 q+1)-n]^{4}}
$$

